\title{
On the kinematics of massive star forming regions: the case of IRAS 17233-3606
}

\author{
S. Leurini ${ }^{1}$, C. Codella ${ }^{2}$, L. Zapata ${ }^{1,3}$, M. T. Beltrán ${ }^{2}$, P. Schilke ${ }^{4}$, and R. Cesaroni ${ }^{2}$ \\ 1 Max-Planck-Institut für Radioastronomie, Auf dem Hügel 69, 53121 Bonn, Germany \\ e-mail: sleurini@mpifr.de \\ 2 INAF - Osservatorio Astrofisico di Arcetri, Largo E. Fermi 5, 50125 Firenze, Italy \\ 3 Centro de Radioastronomía y Astrofísica, Universidad Nacional Autónoma de México, Morelia 58090, Mexico \\ ${ }^{4}$ Physikalisches Institut, Universität zu Köln, Zülpicher Str. 77, 50937 Köln, Germany
}

Received 23 November 2010 / Accepted 29 March 2011

\begin{abstract}
Context. Direct observations of accretion disks around high-mass young stellar objects would help to discriminate between different models of formation of massive stars. However, given the complexity of massive star forming regions, such studies are still limited in number. Additionally, there is still no general consensus on the molecular tracers to be used for such investigations.

Aims. Because of its close distance and high luminosity, IRAS 17233-3606 is a potential laboratory to search for traces of rotation in the inner gas around the protostar(s). Therefore, we selected the source for a detailed analysis of its molecular emission at $230 \mathrm{GHz}$ with the SMA.

Methods. We systematically investigated the velocity fields of transitions in the SMA spectra which are not affected by overlap with other transitions, and searched for coherent velocity gradients to compare them to the distribution of outflows in the region. Beside $\mathrm{CO}$ emission we also used high-angular $\mathrm{H}_{2}$ images to trace the outflow motions driven by the IRAS 17233-3606 cluster.

Results. We find linear velocity gradients in many transitions of the same molecular species and in several molecules. We report the first detection of $\mathrm{HNCO}$ in molecular outflows from massive YSOs. We discuss the $\mathrm{CH}_{3} \mathrm{CN}$ velocity gradient taking into account various scenarios: rotation, presence of multiple unresolved sources with different velocities, and outflow(s). Although other interpretations cannot be ruled out, we propose that the $\mathrm{CH}_{3} \mathrm{CN}$ emission might be affected by the outflows of the region. Higher angular observations are needed to discriminate between the different scenarios.

Conclusions. The present observations, with the possible association of $\mathrm{CH}_{3} \mathrm{CN}$ with outflows in a few thousands $\mathrm{AU}$ around the YSOs' cluster, (i) question the choice of the tracer to probe rotating structures, and (ii) show the importance of the use of $\mathrm{H}_{2}$ images for detailed studies of kinematics.
\end{abstract}

Key words. stars: formation - ISM: molecules - stars: individual: IRAS 17233-3606

\section{Introduction}

Our understanding of the formation of massive stars has largely improved in recent years. From a theoretical point of view, it has been demonstrated, through 2-D and 3-D radiation hydrodynamic simulations (Krumholz et al. 2009; Kuiper et al. 2010), that radiation pressure is not a barrier to form stars with masses $>8 M_{\odot}$ and spectral type $\mathrm{O}$ and $\mathrm{B}$ and that they form through disk accretion. In fact, the two main theoretical scenarios proposed to explain the formation of massive stars are accretionbased mechanisms: (1) the core accretion model (McKee \& Tan 2002, 2003), where a massive star forms from a massive core fragmented from the natal molecular cloud; and (2) the competitive accretion model (Bonnell et al. 2007), where a molecular cloud initially fragments into low-mass cores, which form stars that compete to accrete mass from a common gas reservoir. Both models predict the existence of protostellar accretion disks around massive young stellar objects (YSOs), and therefore the presence of molecular outflows, although the properties of both disks and outflows could be different. In fact, the competitive accretion model suggests that massive stars should always form in densely clustered environments, and so the circumstellar disks could be severely perturbed by interaction with stellar companions.
Over the last years, indirect evidence of the existence of accretion disks have been provided by observations of phenomena such as collimated outflows and jets with properties similar to those originating from low-mass YSOs, thus implying a similar formation mechanism for low- and high-mass stars. However, direct observations of accretion disks in high-mass star-forming regions are still limited to few cases, mostly around YSOs with masses $\leq 20 M_{\odot}$ and luminosities $\leq 10^{4} L_{\odot}$ (e.g., Kraus et al. 2010). The situation is less clear for more luminous objects (Cesaroni et al. 2007), although recently Zapata et al. $(2009,2010)$ suggested the presence of a Keplerian infalling ring around a central object of at least $60 M_{\odot}$. This casts some doubts on the existence of disks around O-type stars as no conclusive evidence for coherent, stable accretion disks around them as been presented so far. The clustered mode of high-mass star formation and the typical large distances of high-mass star forming regions challenge direct observation of accretion disks. In addition, it is still unclear which molecular lines are best suited for such studies. They should have low optical depths, and trace only the hot gas around the central protostar, and not its envelope. In the past, molecules such as $\mathrm{NH}_{3}, \mathrm{CH}_{3} \mathrm{CN}, \mathrm{HCOOCH}_{3}$, and $\mathrm{C}^{34} \mathrm{~S}$ have been proposed for this purpose (i.e., Zhang et al. 1998; Cesaroni et al. 1997, 1999; Beuther et al. 2004, 2009). 
However, there is still no general consensus on the molecular tracer to use (i.e., Beuther et al. 2005b).

IRAS 17233-3606 (hereafter IRAS 17233) is one of the best laboratories to study the problem of massive star formation. Faúndez et al. (2004) reported a luminosity of $1.7 \times 10^{4} L_{\odot}$ obtained by integrating the spectral energy distribution and assuming $d=0.8 \mathrm{kpc}$. Distances between $700 \mathrm{pc}$ and $2.2 \mathrm{kpc}$ are reported in the literature (i.e., Miettinen et al. 2006; Forster \& Caswell 1989). However, the latter is based on maser emission lines. Using the rotation curve determined by Brand \& Blitz (1993) and velocities based on thermal lines (Bronfman et al. 1996; MacLeod et al. 1998; Miettinen et al. 2006; Leurini et al. 2011), the kinematical distance of IRAS 17233 is constrained to $\leq 1 \mathrm{kpc}$. The far distance $(\sim 15 \mathrm{kpc})$ is unlikely because of the source extremely bright continuum and line emission at practically all observed wavelengths, which would indicate exceedingly high luminosities if the source were at the far distance. In the following, we assume a distance of $1 \mathrm{kpc}$ for IRAS 17233.

Thanks to combined high angular resolution observations of different tracers, Leurini et al. (2009) found evidence for multiple outflows driven by different hyper-compact $\mathrm{H}_{\text {II }}$ (HCH II) regions resolved at milliarcsec-scale with VLA by Zapata et al. (2008) (see Fig. 1). Their parameters are typical of massive YSOs (early B-type ZAMS stars) and in agreement with the measured bolometric luminosity of the source. Beuther et al. (2009) found evidence for a large-scale rotating structure detected in high excitation lines of $\mathrm{NH}_{3}$ along the ESE-WNW direction. On the other hand, the high spatial-resolution maps in $\mathrm{CO}$ and $\mathrm{H}_{2}$ by Leurini et al. (2009) indicated a compact bipolar outflow located along the NW-SE, thus questioning the interpretation of the $\mathrm{NH}_{3}$ gradient as a rotating structure.

We present here observations of the massive star forming region IRAS 17233 in several molecular tracers around $230 \mathrm{GHz}$ taken with the Submillimeter Array. The idea of this study is to analyse the spatial distribution and velocity field of different lines, compare them to the distribution of outflows in the region, and verify whether we find evidence for rotating structures perpendicular to the outflows or not. Given the large bandwidth of SMA spectra $(\sim 4 \mathrm{GHz})$ and the richness of the molecular spectrum of the source, such observations allow us to study the distribution of several lines, and not only of the "best" disk tracer candidates.

\section{Molecular outflows from the YSOs in IRAS 17233-3606}

In order to investigate the presence of possible accretion disks around the protostars in the inner region of IRAS 17233, a detailed knowledge of the molecular outflows in the area is needed. In Leurini et al. (2009) we analysed the emission of CO isotopologues observed simultaneously to the data presented in this paper (see Sect. 3) and compared their distribution to high-spatial resolution $\mathrm{H}_{2}$ observations. The main results of that study can be summarised in the following points (see Fig. 1):

1. The data reveal a clumpy extended structure with well separated blue- and red-shifted emission, and an overall structure roughly aligned along the $\mathrm{N}-\mathrm{S}$ direction. The outflow is associated with extremely high velocities, up to $\sim-200$ and $+120 \mathrm{~km} \mathrm{~s}^{-1}$ with respect to the ambient LSR velocity.

2. Using high angular resolution maps of $\mathrm{H}_{2}$, cm-continuum, and maser emission, multiple outflows can be distinguished in the region. One (called here OF1) is compact $\left(\sim 5^{\prime \prime}-10^{\prime \prime}\right)$ and located along the NW-SE direction, as detected in $\mathrm{CO}$
Table 1. Observational parameters.

\begin{tabular}{ccccc}
\hline \hline & $\begin{array}{c}\text { HPBW } \\
\left({ }^{\prime \prime}\right)\end{array}$ & $\begin{array}{c}\text { PA } \\
\left({ }^{\circ}\right)\end{array}$ & $\begin{array}{c}\Delta v \\
\left(\mathrm{~km} \mathrm{~s}^{-1}\right)\end{array}$ & $\begin{array}{c}\mathrm{rms} \\
\left(\mathrm{Jy} \mathrm{beam}^{-1}\right)\end{array}$ \\
\hline cont. & $4^{\prime \prime} 9 \times 1^{\prime \prime \prime} 8$ & $\sim 29^{\circ}$ & & 0.04 \\
LSB & $5^{\prime \prime} .4 \times 1^{\prime \prime} .9$ & $\sim 29^{\circ}$ & 0.5 & 0.1 \\
USB & $4^{\prime \prime} 9 \times 1 . .8$ & $\sim 29^{\circ}$ & 0.5 & 0.1 \\
\hline
\end{tabular}

and SO: it shows extremely high-velocity red-shifted emission and it is associated with a jet traced by $\mathrm{H}_{2}$ emission as well as with red and blue $\mathrm{H}_{2} \mathrm{O}$ maser spots. The extended emission along the $\mathrm{N}-\mathrm{S}$ direction is probably due to multiple outflows. The picture derived by connecting the position of the $\mathrm{CO}$ high velocity clumps suggests two possible main axes, here labelled as OF2 and OF3 and drawn in Fig. 1 by dashed lines. Interestingly, OF2 is associated with a counterpart on smaller scales $\left(\sim 5^{\prime \prime}\right.$, corresponding to $\sim 5000 \mathrm{AU}$ at $D=1 \mathrm{kpc}$ ) traced by red- and blue-shifted $\mathrm{OH}$ masers (Fig. 1b). In addition, another possible outflow direction is indicated in Fig. 1 by the dotted line.

3. The powering sources of the flows remain unidentified, as they are centred on a region where $4 \mathrm{HCH}$ II regions (VLA 2a, b, c and d, see Fig. 1b and 2) have been observed on a sub-arcsec scale.

\section{Observations}

We observed IRAS 17233 with the SMA interferometer on April 10,2007 in the compact configuration with seven antennas. The receivers operated in a double-sideband mode with an IF band of 4-6 GHz so that the upper and lower sideband (USB and LSB, respectively) were separated by $10 \mathrm{GHz}$. The central frequencies of the upper and lower sideband were 220.4 and $230.4 \mathrm{GHz}$, respectively.

The observations presented in this study were performed simultaneously to those of CO discussed by Leurini et al. (2009). For details on the weather conditions, calibration, and reduction of the dataset we refer to their Sect. 2. We checked the absolute positional uncertainty on the quasar $3 \mathrm{C} 273$, our phase calibrator, and found it to be $<0$.' 8 for the right ascension and $<0$ ' 5 for the declination.

The resulting synthesised beams, the velocity resolution used in the analysis and the typical r.m.s of the maps are given in Table 1 . The centre of the maps is at $\alpha_{2000}=$ $17^{\mathrm{h}} 26^{\mathrm{m}} 41^{\mathrm{s}} \cdot 757, \delta_{2000}=-36^{\circ} 09^{\prime} 00^{\prime} 50$. The conversion factor from flux density to brightness temperature in the synthesised beam is $\sim 2.25 \mathrm{~K}(\mathrm{Jy} / \text { beam })^{-1}$ for the lower side band, and $\sim 2.53 \mathrm{~K}(\mathrm{Jy} / \text { beam })^{-1}$ for the upper side band data.

\section{Observational results}

\subsection{Continuum emission}

The continuum emission of the region at $1.3 \mathrm{~mm}$ is shown in Fig. 2. This was derived using only the upper side band data, which have a lower number of lines than the lower side band data and therefore line subtraction is less problematic. The peak of the emission is found close to the cluster of four compact sources detected at cm-wavelengths by Zapata et al. (2008). This also corresponds to the so-called "maser zone", where strong $\mathrm{H}_{2} \mathrm{O}, \mathrm{CH}_{3} \mathrm{OH}$, and $\mathrm{OH}$ masers are detected (e.g., Caswell et al. 1980; Fix et al. 1982; Menten 1991, see Fig. 1b). Weak $(\sim 3 \sigma)$ emission is also detected from the $\mathrm{H}_{\text {II }}$ region on the east of the 

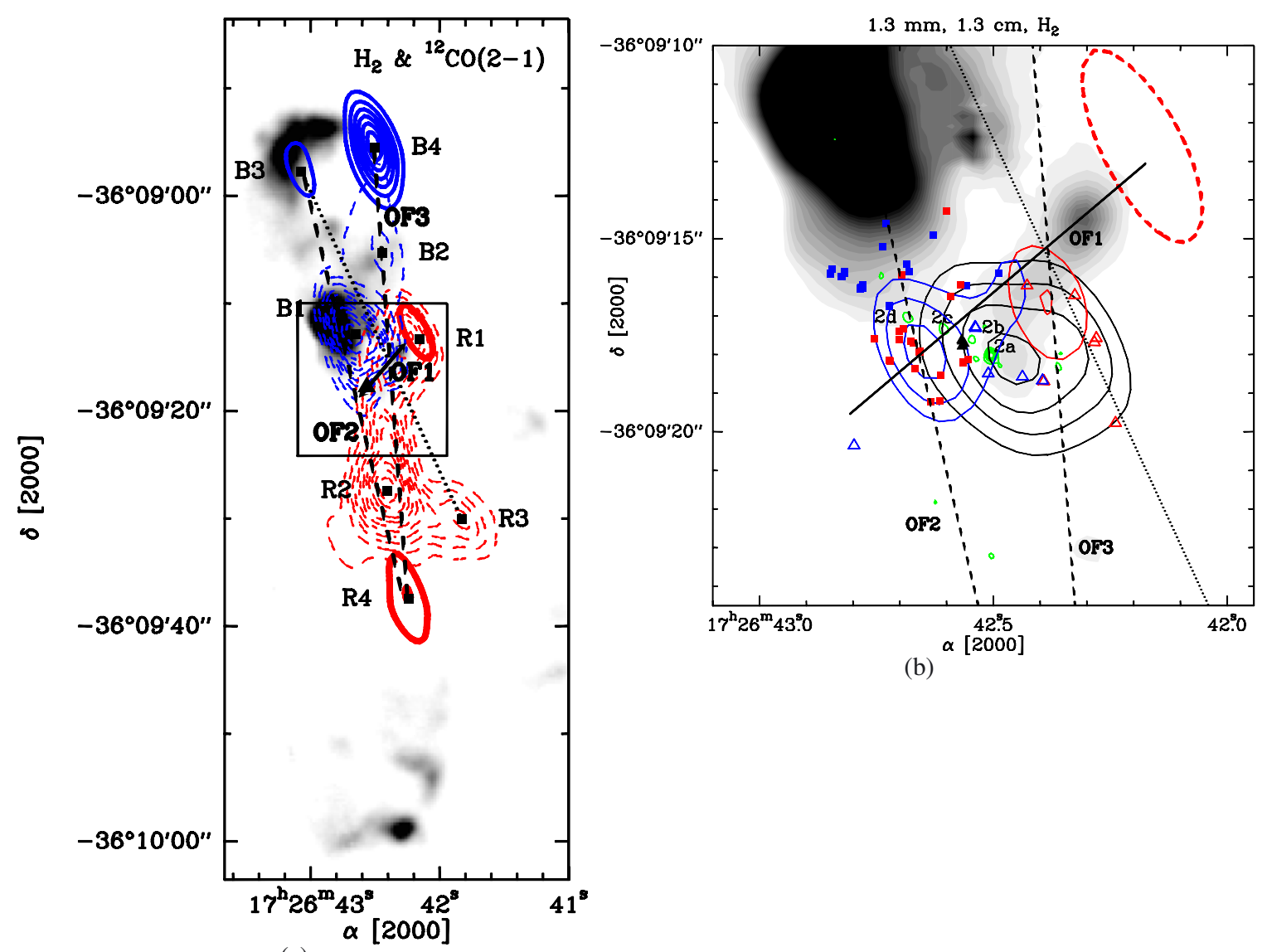

(b)

(a)

Fig. 1. Left panel: integrated emission of the blue- and red-shifted wings in the $\mathrm{CO}(2-1)$ line overlaid on the $\mathrm{H}_{2}$ emission at $2.12 \mu \mathrm{m}$ (grey scale). The solid contours show the EHV blue- $\left(v=[-200,-130] \mathrm{km} \mathrm{s}^{-1}\right)$ and red-shifted emission $\left(v=[90,120] \mathrm{km} \mathrm{s}^{-1}\right)$; the dashed contours mark the HV blue- $\left(v=[-130,-25] \mathrm{km} \mathrm{s}^{-1}\right)$ and red-shifted emission $\left(v=[16,50] \mathrm{km} \mathrm{s}^{-1}\right)$. The dashed lines and the solid arrow outline the possible molecular outflows identified in CO (OF1, OF2, OF3, see discussion in Sect. 2); the dotted line marks the alternative direction of the OF2 outflow. The central box outlines the region mapped in the right panel. The squares mark the positions of the red- and blue-shifted $\mathrm{CO}(2-1)$ peaks. Right panel: continuum emission at $1.3 \mathrm{~mm}$ from the maser zone in IRAS 17233 (solid black contours from 50\% of peak emission in step of 15\%, see Sect. 4.1). Overlaid on the map are the positions of the $\mathrm{OH}$ (blue and red squares, Fish et al. 2005), $\mathrm{CH}_{3} \mathrm{OH}$ (black triangles, Walsh et al. 1998) and water (blue and red open triangles, Zapata et al. 2008) masers. The green contour levels show the continuum emission at $1.3 \mathrm{~cm}$ (from $1.4 \mathrm{mJy}_{\text {beam }}{ }^{-1}, 3 \sigma$, in step of $1.4 \mathrm{mJy}_{\text {beam }}^{-1}$; Zapata et al. 2008), while the grey scale map is the $\mathrm{H}_{2}$ emission. The solid blue and red contours represent the $\mathrm{SO}\left(5_{6}-4_{5}\right)$ blue- and red-shifted emission (blue: from 80 to $104 \mathrm{Jy} \mathrm{km} \mathrm{s}^{-1}$ beam $^{-1}$ in step of $10 \mathrm{Jy} \mathrm{km} \mathrm{s}^{-1}$ beam $^{-1}$; red: 10 and $12 \mathrm{Jy} \mathrm{kms}^{-1}$ beam ${ }^{-1}$ ). The dashed red contour shows the EHV red-shifted $\mathrm{CO}(2-1)$ emission. OF1, OF2 and OF3 are marked as in left panel.

maser zone, already mapped at cm-wavelengths by several authors (e.g., Hughes \& MacLeod 1993; Walsh et al. 1998).

We performed a 2-D Gaussian fit of the $1.3 \mathrm{~mm}$ continuum emission, and determined its peak to be at $\alpha_{\mathrm{J} 2000}=$ $17^{\mathrm{h}} 26^{\mathrm{m}} 42.455^{\mathrm{s}}, \delta_{\mathrm{J} 2000}=-36^{\circ} 09^{\prime} 18.047^{\prime \prime}$ with positional errors of 0 .' $^{\prime} 08$. The peak flux $\left(F_{v}\right)$ is $\sim 2.09 \pm 0.07 \mathrm{Jy} \mathrm{beam}^{-1}$, the integrated flux $\left(S_{v}\right) 7.20 \mathrm{Jy}$. However, given the richness of the molecular spectrum of the region at this frequency, the continuum emission may have been overestimated because of line contamination. The inferred source size is $6 . .1 \pm 0 ., 2 \times 5^{\prime \prime} .1 \pm 0.2$ with a PA of $64^{\circ} .2 \pm 7^{\circ} .5$, corresponding to a deconvolved size of $5^{\prime \prime} .3 \times 2$ '. 7 , and a PA of $-77^{\circ} .2$ (see Table 3 ).

The peak of the mm continuum emission does not correspond to any of the compact sources detected by Zapata et al. (2008) at $1.3 \mathrm{~cm}$, but it is shifted to the west. VLA 2a, b, c and d are all within $\sim 2^{\prime \prime}$ from the peak of the mm continuum emission. However, the displacement between the mm continuum peak and the strongest $\mathrm{cm}$ source VLA $2 \mathrm{a}$ is only $\sim\left(0{ }^{\prime} 6,0{ }^{\prime}(0)\right.$, while the offset from the $\mathrm{CH}_{3} \mathrm{OH}$ maser spots (whose uncertainty in the absolute position is $\sim 1^{\prime \prime}$, Walsh et al. 1998) is $\sim\left(1^{\prime \prime} 4,0,0^{\prime} 3\right)$. Given the uncertainty in the absolute position of our SMA data $\left(\$ 0{ }^{\prime} 8\right)$, this may not be significative.

We can calculate beam-averaged gas column density and mass using the equations given by Beuther et al. (2002) corrected in the erratum by Beuther et al. (2005a). We assume the authors' default values for the grain size $(0.1 \mu \mathrm{m})$, grain mass density $\left(3 \mathrm{~g} \mathrm{~cm}^{-3}\right)$, gas-to-dust ratio (100) and a typical dust temperatures of $200 \mathrm{~K}$ for hot cores. For the grain emissivity, we used $Q_{v}=7.5 \times 10^{-4}(125 \mu \mathrm{m} / \lambda)^{\beta}, \beta=1.6$, as given by Beuther et al. (2005a). We derived a beam-averaged $\mathrm{H}_{2}$ column density of $1.0 \times 10^{24} \mathrm{~cm}^{-2}$, and a mass of $12 M_{\odot}$. However, the mass and column density derived in this fashion are affected by the uncertainties in the peak and integrated flux determinations due to line contamination. Based on unpublished SMA observations at $217 \mathrm{GHz}$ in the same configuration of the array as for the current data (Leurini et al., in prep.), we derived a second independent measurement of $S_{v}$ and $F_{v}$ at a wavelength close to that presented here. The peak flux at $217 \mathrm{GHz}$ is estimated to be $2.10 \pm 0.04 \mathrm{Jy}$ beam $^{-1}$ (on a beam of 3 ". $31 \times 22^{\prime \prime} 54$ ), while the integrated intensity of the source is $6.69 \mathrm{Jy}$. These values are 


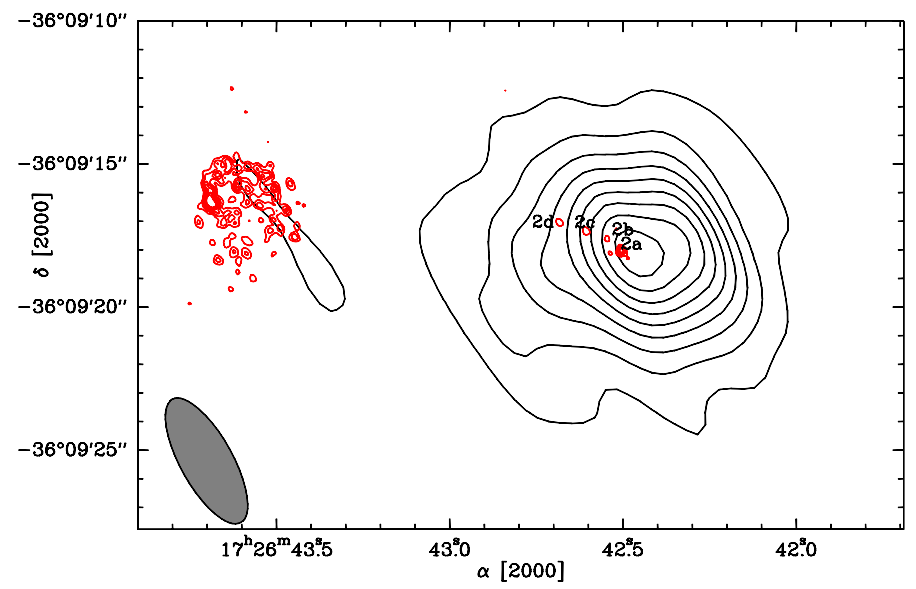

Fig. 2. Continuum emission at $1.3 \mathrm{~mm}$ (black contours) and $1.3 \mathrm{~cm}$ (red contours) of IRAS 17233. Contours are from $2 \mathrm{mJy}^{-1}$ beam $^{-1}(5 \sigma)$ in step of $2 \mathrm{mJy}^{\mathrm{beam}}{ }^{-1}$ ( $1.3 \mathrm{~cm}$ emission), and from $0.12 \mathrm{Jy} \mathrm{beam}^{-1}(3 \sigma)$ in steps of $0.24 \mathrm{Jy}_{\text {beam }}^{-1}$ (1.3 mm emission). The four HCH II VLA 2a, b, c and d identified by Zapata et al. (2008) are also labelled.

very close to those derived at $230 \mathrm{GHz}$, with an uncertainty of less than $10 \%$ in the peak flux (but on a different beam) and on the integrated intensity. Therefore, the uncertainties in the estimates of the mass and of the column density are dominated by the uncertainty in the dust temperature.

\subsection{Line emission}

The molecular spectrum associated with the peak of the $1.3 \mathrm{~mm}$ continuum emission is very rich in lines, and typical of hot cores in massive YSOs. The LSB spectrum of the source (Fig. 3) is dominated by the $\mathrm{CH}_{3} \mathrm{CN}\left(12_{K}-11_{K}\right)$ band: transitions up to $K=$ 9 are detected, together with lines from the $v_{8}=1$ state (up to $K=7$ ), and from the $\mathrm{CH}_{3}^{13} \mathrm{CN}$ isotopologue (up to $K=6$ ). This points to high excitation conditions since the lower level energies of these lines range up to $840 \mathrm{~K}$. The detection frequency of lines in the USB spectrum is less than for the LSB band, and the band is dominated by the $\mathrm{CO}(2-1)$ transition.

Given the complexity of the region and the richness of the spectrum, line identification in molecular spectra of hot cores is challenging. In the analysis of the spectrum of IRAS 17233, we made use of the XCLASS program ${ }^{1}$. The method used in the program is described by Comito et al. (2005) (and references therein), and it is based on a simultaneous fit of all lines in a spectrum under the LTE approximation. The model takes into account the beam dilution, the line opacity, and the line blending, and produces a synthetic spectrum of the source for given physical parameters $\left(T_{\text {kin }}, N_{\text {species }}\right.$, source size $)$. The molecular spectroscopic parameters are taken from the CDMS catalogue (Müller et al. 2001, 2005) and from the molecular spectroscopic database of the Jet Propulsion Laboratory (JPL, see Pickett et al. 1998).

Although this approach delivers physical quantities such us rotational temperature, column density and source size for each of the molecular species included in the model, we believe that the complexity of the region is too high to infer meaningful results with such a simple approach. The analysis of the hot core chemistry of the source is beyond the scope of this paper and will

\footnotetext{
${ }^{1}$ http://www . astro.uni-koeln.de/projects/schilke/ XCLASS
}

be treated in a dedicated study. Nevertheless, a LTE synthetic spectrum, obtained with plausible input values for the physics of the gas of the inner region around the protostars, is fundamental for a correct identification of spectral features, since it predicts intensities for any transition of a given molecular species at all known frequencies. Based on the line profiles, and on the distribution of several transitions which show extended emission (see Sect. 5.1), we assumed two components inside the beam, the first with a compact $\left(\leq 2^{\prime \prime}\right)$ source with a temperature of $\sim 200 \mathrm{~K}$ and a line width of $4 \mathrm{~km} \mathrm{~s}^{-1}$, and a second more extended $\left(\sim 10^{\prime \prime}\right)$ source with lower temperature $(50-70 \mathrm{~K})$ and broader line width $\left(\sim 10 \mathrm{~km} \mathrm{~s}^{-1}\right)$. Table 2 lists all transitions identified in the LSB and USB spectra of IRAS 17233 towards the peak of the mm continuum emission, which are analysed in this paper, and their line parameters obtained by using Gaussian profiles.

\section{Data analysis}

The frequency setup of our observations covers the $\mathrm{CH}_{3} \mathrm{CN}$ $\left(12_{K}-11_{K}\right)$ band, used in the past to trace disks and/or toroids around massive YSOs, as well as species which have been detected in molecular outflows from low- and high-mass protostars (i.e., $\mathrm{H}_{2}^{13} \mathrm{CO}, \mathrm{CH}_{3} \mathrm{OH}$, OCS). In addition, several high energy transitions are detected, which are potentially good candidates to search for tracers of rotation.

To study the velocity field traced by different molecular features, we derived maps of the zero-th and first moments of different transitions. We limited our analysis to lines which are not affected by overlap with other transitions. This was verified with the help of our LTE model (see Sect. 4.2). However, since several spectral features remain unidentified, it is possible that lines used to derive the distribution of the zero-th and first moments are affected by emission from species not included in our model.

Table 2 lists all transitions identified in the LSB and USB spectra of IRAS 17233 towards the peak of the mm continuum emission and used to derive the zero-th and first moment maps. Part of the LSB spectrum towards the peak of the continuum is shown in Fig. 3 as example of the complexity of the source. Figures 4-10 and 13-14 show the derived distributions of the zero-th order and first moments computed over a region where the signal-to-noise ratio in the line data cubes is larger than $5 \sigma$ (corresponding to $0.5 \mathrm{Jy} \mathrm{beam}^{-1}$ ).

\subsection{Spatial distribution}

As a first step to investigate the association of given transitions with the molecular outflows in the region, with potential accretion disks or with the envelope around protostars, we derived the integrated intensity of different lines to study their spatial distribution. In Figs. 4-7 we present the integrated intensity distribution of selected transitions (Table 2). All the maps are elongated along the PA of the beam of the observations. For all maps, we performed a 2-D Gaussian fit to infer the $F W H M$ size and peak position of the integrated intensity distributions. The results are listed in Table 3. All emission maps are resolved with our current resolution, except those of the $\mathrm{HNCO}\left(10_{3}-9_{3}\right), \mathrm{HC}_{3} \mathrm{~N}\left(24_{2}-23_{2}\right) v_{7}=2, \mathrm{CH}_{3}^{13} \mathrm{CN}\left(12_{2}-11_{2}\right)$, and $\mathrm{CH}_{3} \mathrm{CN}\left(12_{2}-11_{2}\right) v_{8}=1$ lines. The fit suggests that the peak of the molecular emission does not coincide with the 1.3 $\mathrm{mm}$ continuum maximum, but it is located slightly to its NE, with a mean offset of $\left.(9)^{\prime} 0,-17^{\prime \prime} .3\right)$ from the centre of the maps, $(0.6,0 . ! 3)$ from the peak of the mm continuum emission. This could indicate that the hot core is associated with the compact $\mathrm{cm}$ source VLA-2a identified by Zapata et al. (2008) (also 
S. Leurini et al.: On the kinematics of massive star forming regions: the case of IRAS 17233-3606

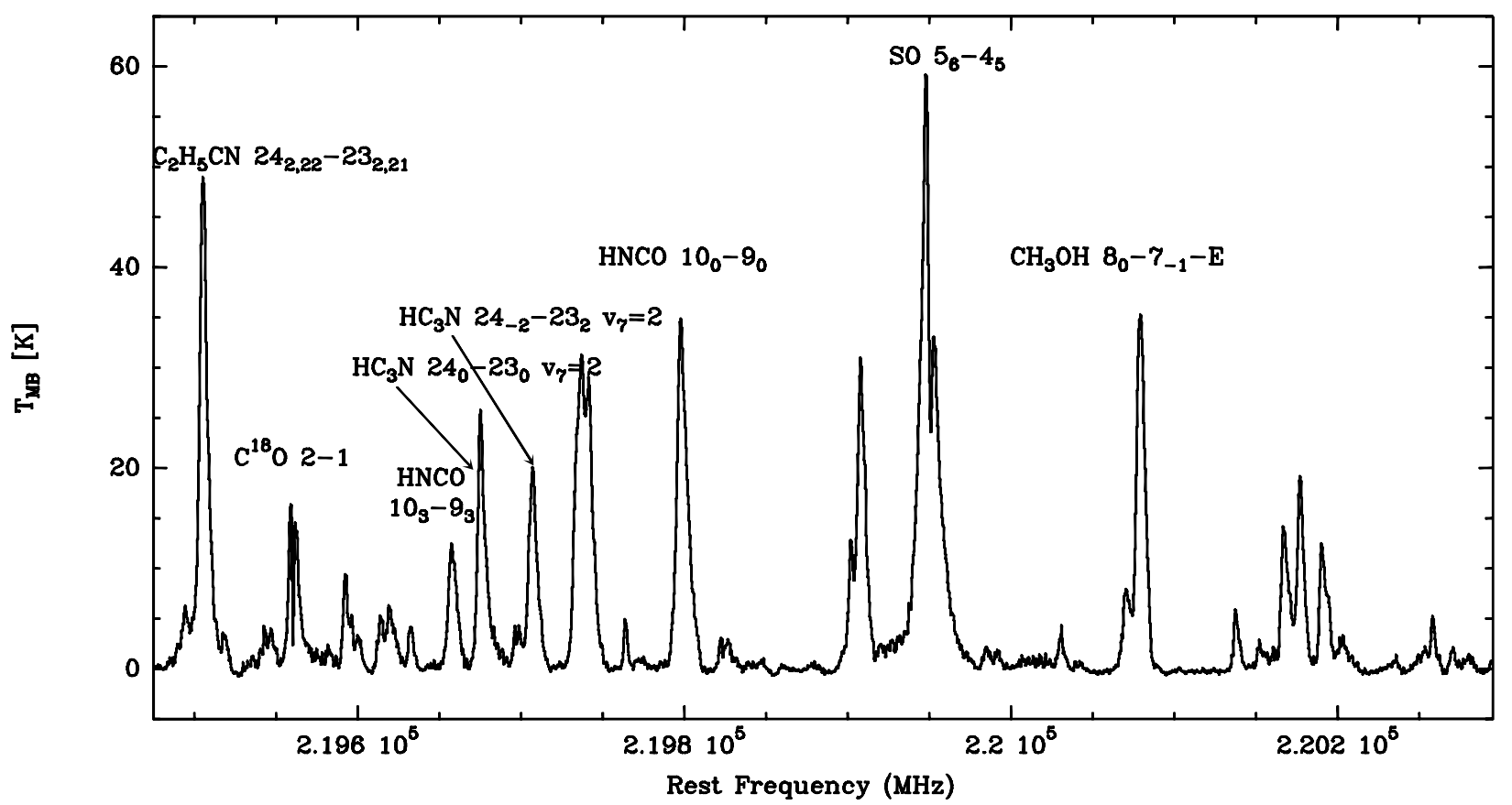

(a)

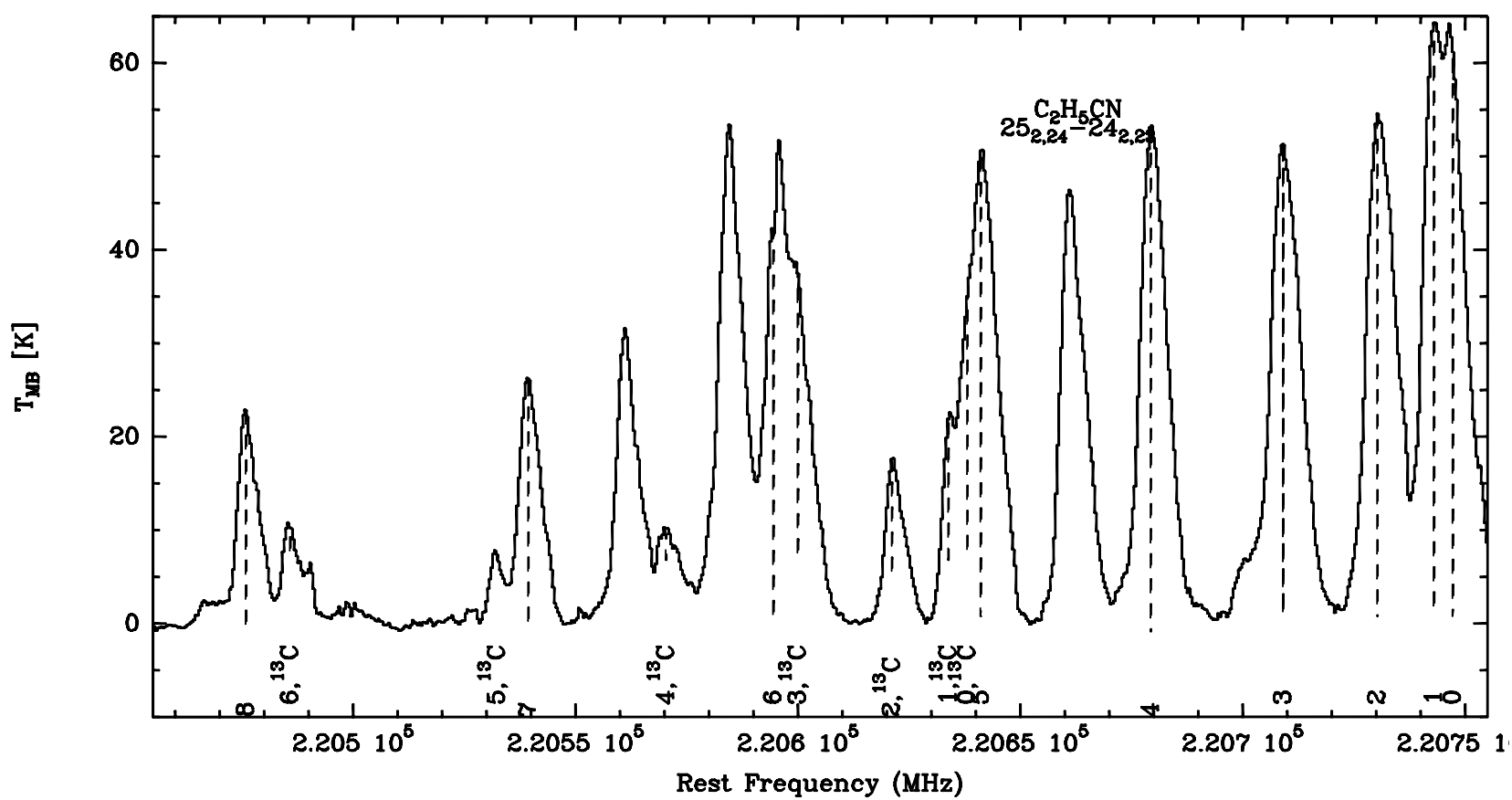

(b)

Fig. 3. Lower side band continuum-subtracted representative spectra of IRAS 17233 at the peak of the $1.3 \mathrm{~mm}$ continuum emission. All transitions used to derive the zero-th and first moment maps of Figs. 4-14 are labelled. For reference, we also mark the $\mathrm{C}^{18} \mathrm{O}(2-1)$ and $\mathrm{SO}\left(5_{6}-5_{5}\right)$ lines (discussed in Leurini et al. 2009), and the ladder of the $\mathrm{CH}_{3} \mathrm{CN}$ (labelled with the corresponding $K$ quantic number) and $\mathrm{CH}_{3}^{13} \mathrm{CN}_{(12-11)}$ lines (labelled with the corresponding $K$ quantic number followed by ${ }^{13} \mathrm{C}$ ).

shifted to the east of the mm continuum), whereas the bulk of continuum is tracing colder material. Opacity effects could play a role in this displacements. Actually, the $\mathrm{CH}_{3} \mathrm{CN}$ spectral pattern shows similar brightness temperatures for lines with $K \leq 5$ (Fig. 3), suggesting non negligible opacities. However, the distribution of less abundant isotopologues (see $\mathrm{O}^{13} \mathrm{CS}$ ) or vibrationally excited transitions $\left(\mathrm{HC}_{3} \mathrm{~N} \mathrm{v}_{7}=2\right.$ lines $)$ seem to peak closer to the continuum peak, but still shifted to the east, near VLA-2a.
Methanol shows extended emission along the axis of the $\mathrm{OF} 2-\mathrm{OF} 3$ outflows, and an elongated structure towards NW, i.e. in the direction of the OF1 red-shifted outflow detected in $\mathrm{H}_{2}$, extremely high velocity $\mathrm{CO}$ and SO (see Fig. 1a) and indicated by an arrow in Figs. 4-7. Interestingly, the occurrence of $\mathrm{CH}_{3} \mathrm{OH}$ emission along the OF1 axis is more evident in the two lower excitation transitions (reported in Table 2). As shown by the spectra in Fig. 3, this is not due to low dynamical range, since all 
Table 2. Spectral features detected in the IRAS 17233 spectrum and analysed in the present paper.

\begin{tabular}{lcrcrr}
\hline \hline \multicolumn{1}{c}{ Transition } & $\begin{array}{c}v \\
{[\mathrm{MHz}]}\end{array}$ & $\begin{array}{r}E_{u} \\
{[\mathrm{~K}]}\end{array}$ & $\begin{array}{r}v_{\mathrm{LSR}} \\
{\left[\mathrm{km} \mathrm{s}^{-1}\right]}\end{array}$ & $\begin{array}{r}\Delta v \\
{\left[\mathrm{~km} \mathrm{~s}^{-1}\right]}\end{array}$ & $\begin{array}{r}\int T \delta v \\
{\left[\mathrm{~K} \mathrm{~km} \mathrm{~s}^{-1}\right]}\end{array}$ \\
\hline $\mathrm{C}_{2} \mathrm{H}_{5} \mathrm{CN}\left(24_{2,22}-23_{2,21}\right)$ & 219505.59 & 106 & $-3.40 \pm 0.11$ & $7.96 \pm 0.29$ & $386.6 \pm 11.0$ \\
$\mathrm{HNCO}\left(10_{3}-9_{3}\right)$ & 219656.77 & 433 & $-5.23 \pm 0.04$ & $8.11 \pm 0.10$ & $100.0 \pm 1.0$ \\
$\mathrm{HC}_{3} \mathrm{~N}\left(24_{0}-23_{0}\right) v_{7}=2$ & 219675.11 & 773 & $-4.29 \pm 0.33$ & $7.45 \pm 0.89$ & $178.8 \pm 16.5$ \\
$\mathrm{HC}_{3} \mathrm{~N}\left(24_{-2}-23_{2}\right) v_{7}=2$ & 219707.35 & 778 & $-3.28 \pm 0.14$ & $8.45 \pm 0.40$ & $163.9 \pm 6.0$ \\
$\mathrm{HNCO}_{1}\left(10_{0}-9_{0}\right)$ & 219798.27 & 58 & $-3.94 \pm 0.01$ & $10.16 \pm 0.03$ & $342.0 \pm 1.0$ \\
$\mathrm{CH}_{3} \mathrm{OH}-E\left(8_{0}-7-1\right)$ & 220078.49 & 97 & $-4.56 \pm 0.12$ & $8.36 \pm 0.32$ & $304.8 \pm 9.2$ \\
$\mathrm{CH}_{3} \mathrm{CN}\left(12_{8}-11_{8}\right)$ & 220475.92 & 525 & $-4.02 \pm 0.05$ & $7.44 \pm 0.12$ & $169.8 .8 \pm 2.2$ \\
$\mathrm{CH}_{3} \mathrm{CN}\left(12_{7}-11_{7}\right)$ & 220539.41 & 419 & $-3.85 \pm 0.43$ & $7.79 \pm 1.07$ & $210.8 \pm 24.2$ \\
$\mathrm{CH}_{3}^{13} \mathrm{CN}\left(12_{2}-11_{2}\right)$ & 220621.14 & 97 & $-4.33 \pm 0.75$ & $6.98 \pm 1.84$ & $123.6 \pm 27.0$ \\
$\mathrm{C}_{2} \mathrm{H}_{5} \mathrm{CN}\left(25_{2,24}-24_{2,23}\right)$ & 220660.92 & 121 & $-4.64 \pm 0.58$ & $8.69 \pm 1.39$ & $407.0 \pm 54.3$ \\
$\mathrm{CH}_{3} \mathrm{CN}\left(12_{4}-11_{4}\right)$ & 220679.32 & 183 & $-4.29 \pm 0.46$ & $9.30 \pm 1.14$ & $515.5 \pm 52.5$ \\
$\mathrm{CH}_{3} \mathrm{CN}\left(12_{2}-11_{2}\right) v_{8}=1$ & 221367.67 & 596 & $-3.63 \pm 0.37$ & $8.14 \pm 0.97$ & $178.5 \pm 17.3$ \\
$\mathrm{CH}_{3} \mathrm{OH}-E\left(8_{-1}-7_{0}\right)$ & 229758.76 & 89 & $-3.22 \pm 0.02$ & $7.94 \pm 0.04$ & $484.0 \pm 2.1$ \\
$\mathrm{O}^{13} \mathrm{CS}(19-18)$ & 230317.53 & 99 & $-2.50 \pm 0.04$ & $6.36 \pm 0.11$ & $118.9 \pm 1.7$ \\
$\mathrm{OCS}(19-18)$ & 231060.99 & 100 & $-3.04 \pm 0.02$ & $10.51 \pm 0.04$ & $574.2 \pm 1.7$ \\
$\mathrm{CH}_{3} \mathrm{OH}-A\left(10_{2}-9_{3}\right)$ & 231281.10 & 165 & $-3.72 \pm 0.03$ & $7.31 \pm 0.08$ & $165.6 \pm 1.3$ \\
\hline
\end{tabular}

Table 3. Result of the 2-D Gaussian fit of the integrated intensity distributions.

\begin{tabular}{|c|c|c|c|c|}
\hline Transition & $\Delta \alpha^{a}$ & $\Delta \delta^{\mathrm{a}}$ & $F W H M$ observed size ${ }^{b}$ & $F W H M$ deconvolved size \\
\hline cont. & $8 . \prime 455$ & $-17^{\prime \prime} .551$ & $6^{\prime \prime} 1 \times 5^{\prime \prime} 1$ & $5^{\prime \prime} .3 \times 2.7$ \\
\hline $\mathrm{C}_{2} \mathrm{H}_{5} \mathrm{CN}\left(24_{2,22}-23_{2,21}\right)$ & 8.'930 & $-17^{\prime \prime} .538$ & $5{ }^{\prime \prime} 9 \times 33^{\prime \prime} 2$ & $2^{\prime \prime} 6 \times 22^{\prime \prime} 2$ \\
\hline $\operatorname{HNCO}\left(10_{3}-9_{3}\right)$ & 8.'987 & $-177^{\prime \prime} 140$ & $5{ }^{\prime \prime} 0 \times 1 . .9$ & \\
\hline $\mathrm{HC}_{3} \mathrm{~N}\left(24_{0}-23_{0}\right) v_{7}=2$ & 8.'941 & $-17^{\prime \prime} .421$ & $5^{\prime \prime} .5 \times 2 . .3$ & $1 . .5 \times 0.4$ \\
\hline $\mathrm{HC}_{3} \mathrm{~N}\left(24_{-2}-23_{2}\right) v_{7}=2$ & 8.' 832 & $-17^{\prime \prime} .475$ & $5^{\prime \prime} .4 \times 22^{\prime \prime} 2$ & \\
\hline $\operatorname{HNCO}\left(10_{0}-9_{0}\right)$ & 8.' 830 & $-17^{\prime \prime} 399$ & $5{ }^{\prime \prime} 9 \times 2.9$ & $2.5 \times 2 . .1$ \\
\hline $\mathrm{CH}_{3} \mathrm{OH}-E\left(8_{0}-7_{-1}\right)$ & 9.'091 & $-17 " .108$ & $5^{\prime \prime} 9 \times 33^{\prime \prime} 9$ & $3^{\prime \prime} 6 \times 22^{\prime \prime} .2$ \\
\hline $\mathrm{CH}_{3} \mathrm{CN}\left(12_{8}-11_{8}\right)$ & 8.' 885 & $-17^{\prime \prime} .245$ & $5^{\prime \prime} .5 \times 2.6$ & $2^{\prime \prime} 0 \times 0 \times 0^{\prime} .4$ \\
\hline $\mathrm{CH}_{3} \mathrm{CN}\left(12_{7}-11_{7}\right)$ & 9.'010 & -17.305 & $5^{\prime \prime} .5 \times 2 . .9$ & $2 . .2 \times 11^{\prime \prime} 0$ \\
\hline $\mathrm{CH}_{3}^{13} \mathrm{CN}\left(12_{2}-11_{2}\right)$ & 8.'900 & $-17^{\prime \prime} .400$ & $5^{\prime \prime} 4 \times 3^{\prime \prime} .0$ & \\
\hline $\mathrm{C}_{2} \mathrm{H}_{5} \mathrm{CN}\left(25_{2,24}-24_{2,23}\right)$ & 9.'076 & $-17^{\prime \prime} .459$ & $5{ }^{\prime \prime} 8 \times 33^{\prime \prime} 1$ & $2 . .6 \times 2 . \prime 0$ \\
\hline $\mathrm{CH}_{3} \mathrm{CN}\left(12_{4}-11_{4}\right)$ & 9.'113 & $-17 . .386$ & $5^{\prime \prime} .9 \times 3 . .8$ & $3^{\prime \prime} .3 \times 2.2$ \\
\hline $\mathrm{CH}_{3} \mathrm{CN}\left(12_{2}-11_{2}\right) v_{8}=1$ & 8.'975 & $-17^{\prime \prime} .375$ & $5^{\prime \prime} .3 \times 22^{\prime \prime} 4$ & \\
\hline $\mathrm{CH}_{3} \mathrm{OH}-E\left(8_{-1}-7_{0}\right)$ & 9.247 & $-16 . ' 994$ & $5^{\prime \prime} .8 \times 4 . .7$ & $4 . .4 \times 2.9$ \\
\hline $\mathrm{O}^{13} \mathrm{CS}(19-18)$ & 8.' 850 & $-17^{\prime \prime} .559$ & $5^{\prime \prime} .2 \times 3^{\prime \prime} .6$ & 3.' $1 \times 11^{\prime \prime} .7$ \\
\hline OCS (19-18) & $9 ! 216$ & $-17{ }^{\prime \prime} 424$ & $5 . \prime 7 \times 44^{\prime \prime} 4$ & $4{ }^{\prime \prime} 0 \times 22^{\prime \prime} 9$ \\
\hline $\mathrm{CH}_{3} \mathrm{OH}-A\left(10_{2}-9_{3}\right)$ & $9 ! 173$ & $-17{ }^{\prime \prime} 082$ & $5.3 \times 33^{\prime \prime} 8$ & $3 . \prime 3 \times 22^{\prime \prime} 0$ \\
\hline
\end{tabular}

Notes. ${ }^{(a)}$ offset from the centre of the maps at $\alpha_{2000}=17^{\mathrm{h}} 26^{\mathrm{m}} 41^{\mathrm{s}} \cdot 757, \delta_{2000}=-36^{\circ} 09^{\prime} 0{ }^{\prime} \cdot 50$ (see Sect. 3). ${ }^{(b)}$ typical errors on the fit are of the order of 0 !' 2 .

methanol lines used in this analysis have a signal-to-noise ratio larger than 40 at the peak of the continuum emission.

Also the OCS emission, and to some extent $\mathrm{O}^{13} \mathrm{CS}$ emission, is elongated in the direction the OF2-OF3 outflows, and shows a wisp of emission in the direction of OF1 (Fig. 5).

Figure 6 shows the zero-th moment maps derived from different lines of $\mathrm{CH}_{3} \mathrm{CN}$. The spatial distribution of the $12_{4}-11_{4}$ line, the lowest $\mathrm{CH}_{3} \mathrm{CN}$ excitation line used in our analysis, extends in the direction of the OF1 jet and along the axis of the OF2-OF3 outflows, while the other transitions are more compact. Also in this case we can exclude a bias due to different signal-to-noise ratios for the different $\mathrm{CH}_{3} \mathrm{CN}$ lines.

The $\mathrm{C}_{2} \mathrm{H}_{5} \mathrm{CN}$ and $\mathrm{HNCO}\left(10_{0}-9_{0}\right)$ line maps are also elongated along the direction of the OF2-OF3 axes, but their emission is more compact than for the other species discussed above.

In conclusion, the spatial distribution of $\mathrm{CH}_{3} \mathrm{OH}, \mathrm{OCS}$, and $\mathrm{CH}_{3} \mathrm{CN}$ emission could be affected by the presence of the outflows. In particular, for $\mathrm{CH}_{3} \mathrm{OH}$ and $\mathrm{CH}_{3} \mathrm{CN}$ we find, besides an elongation along the OF2-OF3 axes, also a good correlation with the direction of the OF1 outflow. Emission at the position
$\mathrm{R} 1$, the peak of the $\mathrm{CO}(2-1)$ red-shifted emission at extremely high velocity in $\mathrm{OF} 1$, is detected from the $\mathrm{CH}_{3} \mathrm{OH}\left(8_{-1}-7_{0}\right)$ and $\left(8_{0}-7_{1}\right)$ lines and the $\mathrm{CH}_{3} \mathrm{CN}\left(12_{4}-11_{4}\right)$ transition. In Sect. 5.2 we will analyse the first moment maps to investigate the kinematics of the gas and better constrain the association of molecular emission with the molecular outflows from the IRAS 17233 cluster.

\subsection{Velocity distribution}

For transitions analysed in Sect. 5.1 with emission larger than 3 beam widths along the minor axis of the beam, we also computed the first moment distribution. This excludes the $\mathrm{HC}_{3} \mathrm{~N}$ transitions from the analysis.

The detected velocity shifts are of the order of $\Delta v \sim 6 \mathrm{~km} \mathrm{~s}^{-1}$, i.e. values larger than velocity resolution of the data cubes $\left(0.5 \mathrm{~km} \mathrm{~s}^{-1}\right)$. All $\mathrm{CH}_{3} \mathrm{CN}$ maps are blue-shifted compared to the systemic velocity $\left(V_{\text {sys }}\right)$ of $-3.4 \mathrm{~km} \mathrm{~s}^{-1}$ (Bronfman et al. 1996). However, there is a large uncertainty in the determination of $V_{\text {sys }}$ (between -3.7 and $-2.5 \mathrm{~km} \mathrm{~s}^{-1}$ from single dish $\mathrm{N}_{2} \mathrm{H}^{+}$and $\mathrm{C}^{17} \mathrm{O}$ 


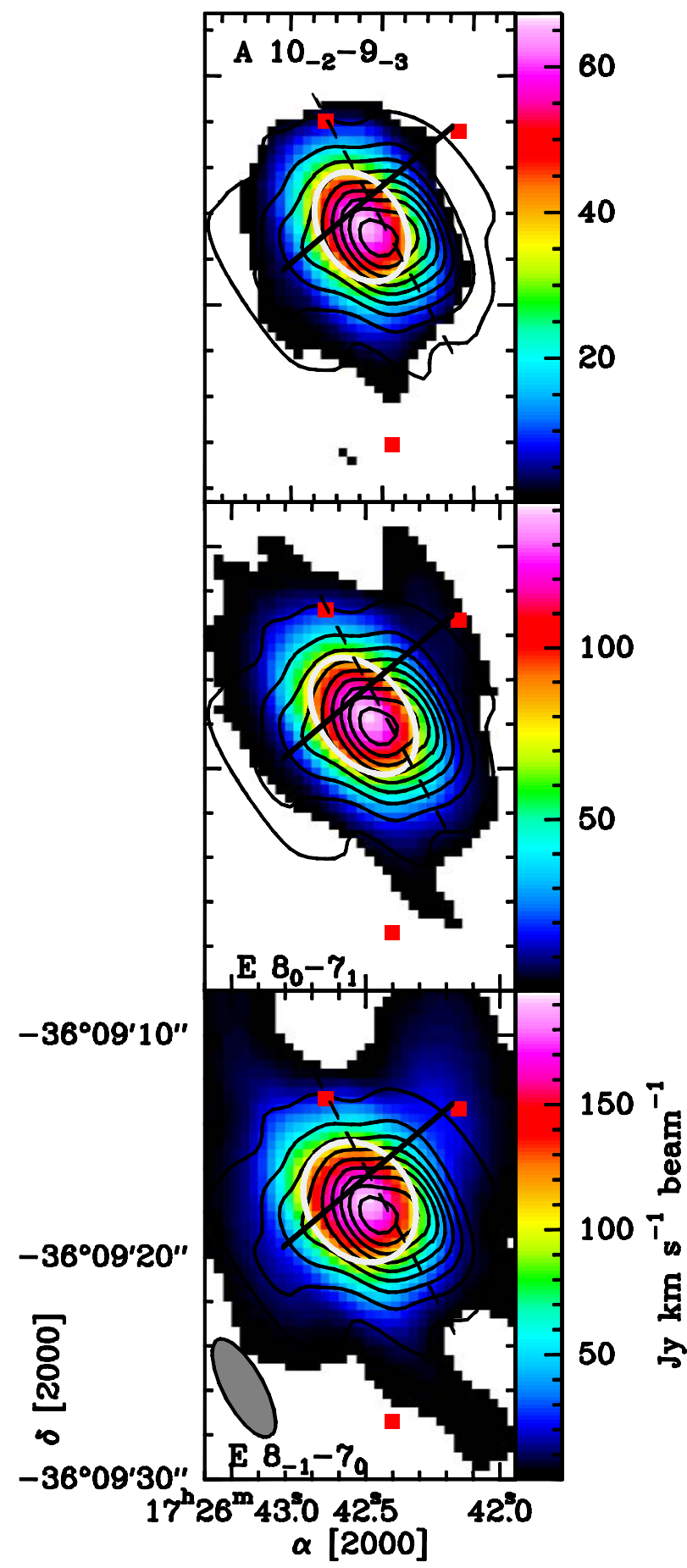

Fig. 4. Integrated emission of selected transitions of $\mathrm{CH}_{3} \mathrm{OH}$ in the region above the $5 \sigma$ detection in the line data cubes $\left(\sim 0.5 \mathrm{Jy} \mathrm{beam}^{-1}\right)$. The black solid contours show the $1.3 \mathrm{~mm}$ continuum emission as in Fig. 2. The solid line shows the axis of OF1, while the dashed line has been chosen, for sake of clarity, to represent the three possible directions of the $\mathrm{N}-\mathrm{S}$ outflows drawn in Fig. 1. The red squares mark the R1, $\mathrm{R} 2$ and $\mathrm{B} 1$ peaks of the $\mathrm{CO}(2-1)$ emission detected towards OF1 and OF2-OF3 (see Fig. 1a). The white ellipse represents the FWHM 2-D Gaussian fit to the data.

observations, Leurini et al. 2011), probably due to the outflow multiplicity in the region.

As suggested by the analysis of the integrated intensity distributions, $\mathrm{CH}_{3} \mathrm{OH}$ clearly presents a velocity gradient along the direction of the OF2-OF3 outflows, with blue-shifted emission

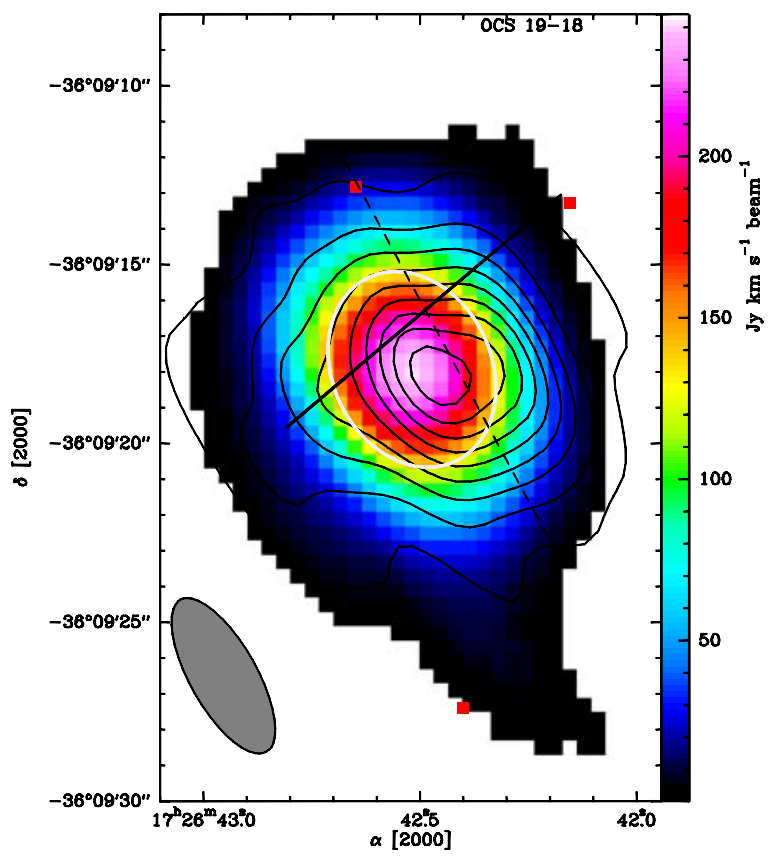

Fig. 5. Integrated emission of the OCS(19-18) line in the region above the $5 \sigma$ detection in the line data cubes $\left(\sim 0.5 \mathrm{Jy} \mathrm{beam}^{-1}\right)$. The black solid contours show the $1.3 \mathrm{~mm}$ continuum emission as in Fig. 2. Symbols are as in Fig. 4.

to the north-east and red-shifted emission to the south-west. Additionally, the $\mathrm{CH}_{3} \mathrm{OH}\left(8_{-1}-7_{0}\right)$ and $\left(8_{0}-7_{1}\right)-E$ lines show red-shifted emission associated with the red lobe of the OF1 jet (see Fig. 1b). A wisp of red-shifted emission towards OF1 is detected also in the $\mathrm{CH}_{3} \mathrm{OH}\left(10_{-2}-9_{-3}\right)$ line although marginally.

For simplicity, we present the first moment distribution of the $\mathrm{CH}_{3} \mathrm{CN}\left(12_{4}-11_{4}\right)$ line in Fig. 9 while the $\mathrm{CH}_{3} \mathrm{CN}\left(12_{7}-11_{7}\right)$ transition is presented in Fig. 10. We see the same behaviour seen in the $\mathrm{CH}_{3} \mathrm{OH}\left(10_{-2}-9_{-3}\right)$ line in the $\left(12_{4}-11_{4}\right)$ transition, the lowest energy analysed line of methyl cyanide: a linear velocity gradient along the E-W axis plus a red-shifted wisp of gas extending to the north-west towards the red-shifted lobe of the OF1 outflow. The $\mathrm{CH}_{3} \mathrm{CN}\left(12_{7}-11_{7}\right)$ line has a more compact emission, and shows a linear velocity gradient along the E-W direction, almost perpendicular to the OF2-OF3 axis. Although not shown here, the velocity field of the $\mathrm{CH}_{3} \mathrm{CN}\left(12_{8}-11_{8}\right)$ line agrees with that of the $\mathrm{CH}_{3} \mathrm{CN}\left(12_{7}-11_{7}\right)$ transition.

In order to further investigate the association of the $\mathrm{CH}_{3} \mathrm{CN}\left(12_{4}-11_{4}\right)$ line with the OF1 outflow, in Fig. 9 we compare its first moment distribution to the $\mathrm{H}_{2}$ distribution and to other tracers of kinematics in the region. The SO blue- and redshifted emission and the blue- and red-shifted $\mathrm{H}_{2} \mathrm{O}$ masers trace the blue and red-shifted lobes of the OF1 outflow (see Fig. 9 and Leurini et al. 2009). If we take into account (i) the EHV OF1 redlobe in $\mathrm{CO}(2-1)$, (ii) the SO red- and blue-shifted emission, and (iii) the water maser spots, then the contribution of OF1 to the kinematics of the $\mathrm{CH}_{3} \mathrm{CN}\left(12_{4}-11_{4}\right)$ transition appears reasonably evident. Furthermore, Fig. 9 also shows red-shifted emission elongated towards the R2 red lobe of OF2-OF3 and blueshifted emission close to the B1 peak of OF2-OF3 as observed in $\mathrm{CO}$ (see Fig. 1). In Fig. 9 we also show the velocity field of the $\mathrm{CH}_{3} \mathrm{CN}\left(12_{3}-11_{3}\right)$ line which has been excluded from our analysis because of weak blending with other features towards the centre position. However, being lower in energy than the $\left(12_{4}-11_{4}\right)$ transition, the $\left(12_{3}-11_{3}\right)$ line has a larger emitting size and can help the interpretation of the kinematics of the other $\mathrm{CH}_{3} \mathrm{CN}$ transitions. In this case, the $\mathrm{N}-\mathrm{W}$ velocity gradient seen 


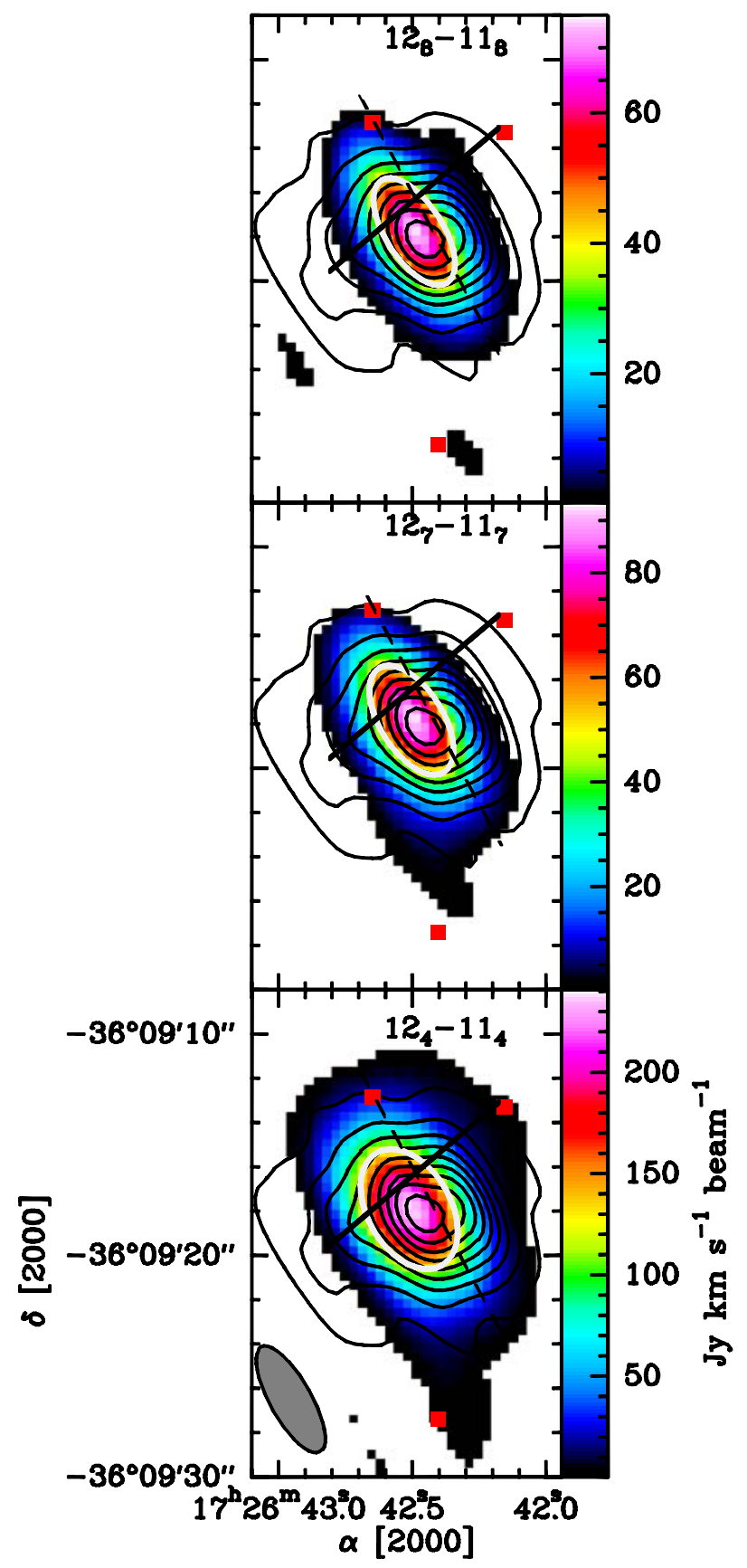

Fig. 6. Integrated emission of selected transitions of $\mathrm{CH}_{3} \mathrm{CN}$ in the region above the $5 \sigma$ detection in the line data cubes $\left(\sim 0.5 \mathrm{Jy}^{\text {beam }}{ }^{-1}\right)$. The black solid contours show the $1.3 \mathrm{~mm}$ continuum emission. Symbols are as in Fig. 4.

in the high $\mathrm{J} \mathrm{CH}_{3} \mathrm{CN}$ transitions and to some extent also in the $\left(12_{4}-11_{4}\right)$ line is less pronounced, while red-shifted emission is clearly detected towards the R1 position in OF1 and towards the red-shifted lobe of the OF2-OF3 outflows.

As an additional check, we derived position-velocity plots along several slits placed to sample all possible directions: the $\mathrm{OF} 1$ axis $\left(\mathrm{PA}-50^{\circ}\right)$, the NE-SW direction (PA $30^{\circ}$, as representative of the three possible directions of the OF2-OF3 outflows), two slits with position angles $60^{\circ}$ and $-20^{\circ}$, the N-S and E-W directions. All six slits are shown in Fig. 9. In Fig. 11 we show the PV diagram along the OF1 axis which shows that $\mathrm{CH}_{3} \mathrm{CN}\left(12_{4}-11_{4}\right)$ emission is spread over a broad range of velocities $\left(\sim 15 \mathrm{~km} \mathrm{~s}^{-1}\right)$. We then extracted spectra of the

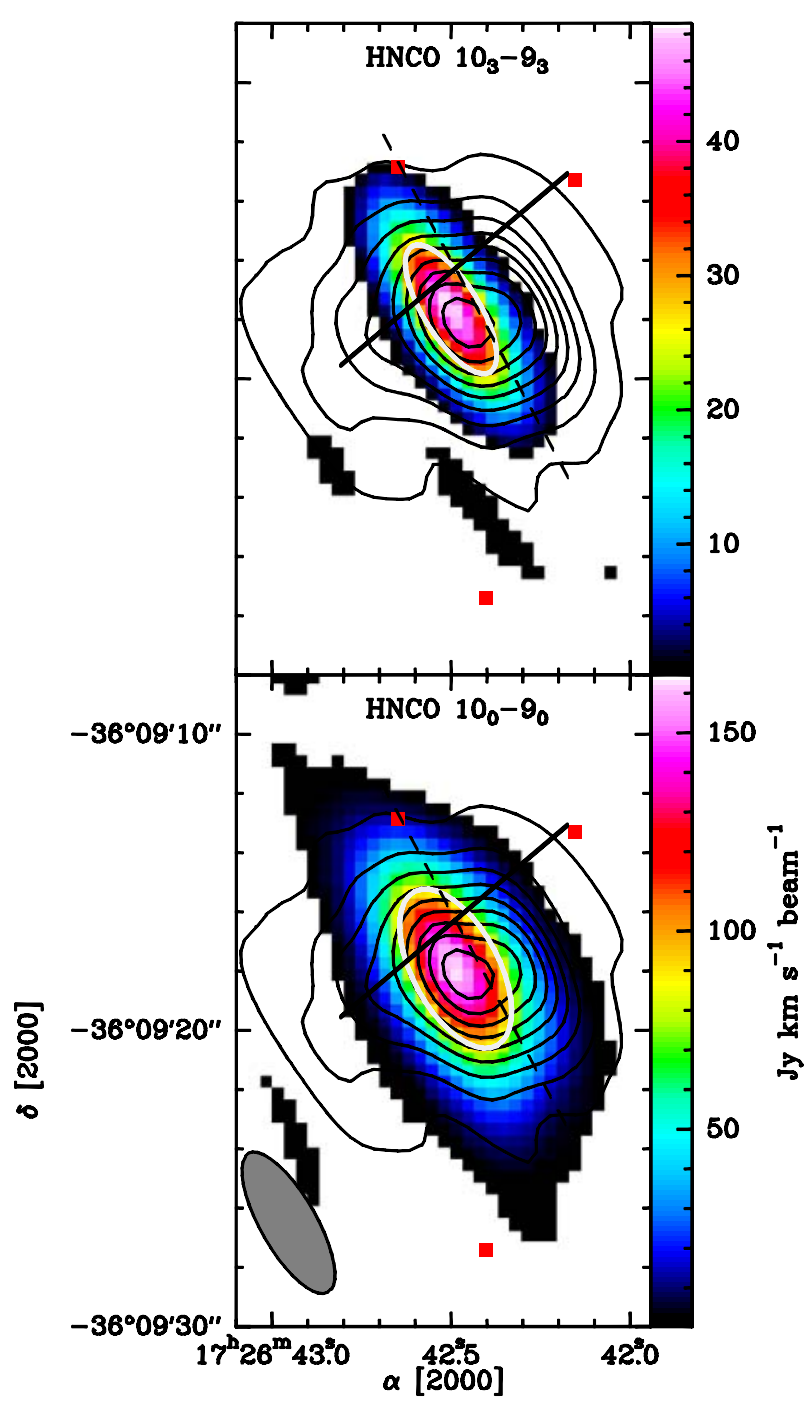

Fig. 7. Integrated emission of selected transitions of $\mathrm{HNCO}$ in the region above the $5 \sigma$ detection in the line data cubes $\left(\sim 0.5 \mathrm{Jy}\right.$ beam $\left.{ }^{-1}\right)$. The black solid contours show the $1.3 \mathrm{~mm}$ continuum emission. Symbols are as in Fig. 4.

$\mathrm{CH}_{3} \mathrm{CN}\left(12_{4}-11_{4}\right)$ transition for each slit, and fitted the line with a Gaussian profile. For each slit, we used a linear fit to reproduce the peak velocity as function of the offset position along the slit. Results are shown in Fig. 12. The largest velocity gradients are detected along the E-W direction $\left(\sim 114 \mathrm{~km} \mathrm{~s}^{-1} \mathrm{pc}^{-1}\right)$ and the OF1 jet $\left(\sim 99 \mathrm{~km} \mathrm{~s}^{-1} \mathrm{pc}^{-1}\right)$. The gradients along OF2$\mathrm{OF} 3$, and the slits at $60^{\circ}$ and $-20^{\circ}$ have similar gradients (32-55 $\mathrm{km} \mathrm{s}^{-1} \mathrm{pc}^{-1}$ ). Only the velocity gradient along the $\mathrm{N}-$ $\mathrm{S}$ direction is negligible, and no fit could be performed for this slit.

Clearly, the velocity gradient along OF1 plays an important role in the kinematics of the inner gas around the protostars. This strongly supports our interpretation that the velocity field of the $\mathrm{CH}_{3} \mathrm{CN}\left(12_{4}-11_{4}\right)$ line is affected by the OF1 outflow. In Sect. 6, we will discuss different scenarios for the velocity gradient detected along the E-W axis.

The velocity field of the OCS(19-18) transition (Fig. 13) is consistent with those of $\mathrm{CH}_{3} \mathrm{OH}$ and of the $\mathrm{CH}_{3} \mathrm{CN}\left(12_{4}-11_{4}\right)$ transition and shows red-shifted emission towards the red peak R1 of OF1 and R2 of OF2-OF3 and blue-shifted emission close to one of the blue peaks of OF2-OF3, B1. O ${ }^{13} \mathrm{CS}$ (not shown 


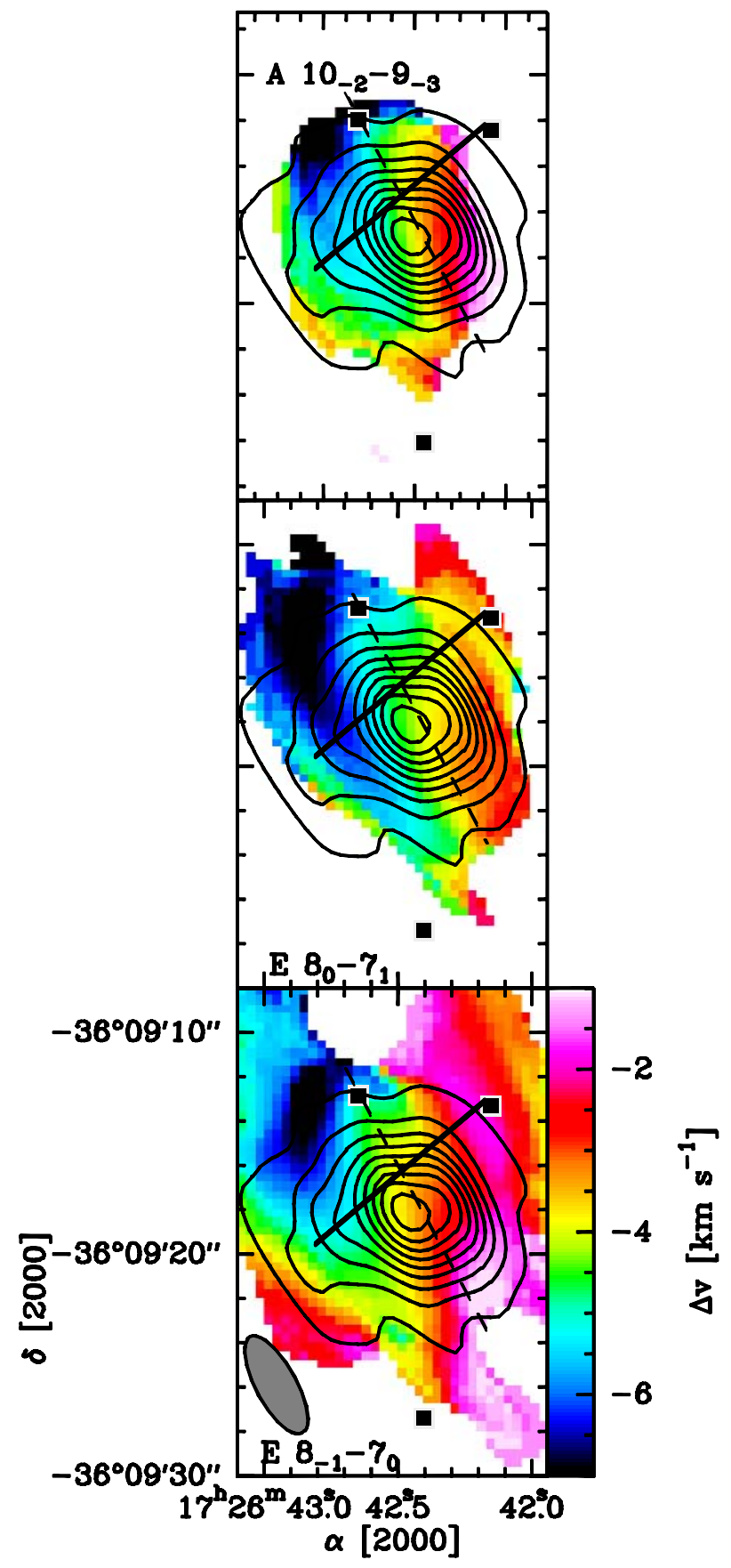

Fig. 8. First moment maps of selected transitions of $\mathrm{CH}_{3} \mathrm{OH}$. The black solid contours show the $1.3 \mathrm{~mm}$ continuum emission. Black squares mark the B1, R1 and R2 positions of Fig. 1a. The other symbols are as in Fig. 4. The systemic velocity of the source lies between -3.4 and $-2.5 \mathrm{~km} \mathrm{~s}^{-1}$ (see Sect. 5.2).

here) is consistent with the picture depicted by high $\mathrm{J} \mathrm{CH}_{3} \mathrm{CN}$ transitions.

Finally, the $\mathrm{HNCO}$ emission shows a velocity gradient in the NE-SW direction along the axis of OF2-OF3 (see Fig. 14). The first moment distribution of the $\mathrm{C}_{2} \mathrm{H}_{5} \mathrm{CN}$ (not shown here) is consistent with those of the high $\mathrm{J} \mathrm{CH}_{3} \mathrm{CN}$ lines.

\section{Discussion}

As seen in Sect. 5.2, different transitions trace different kinematics. On the base of their first moment maps we can distinguish three cases:
1. transitions with a velocity gradient along the NE-SW direction (OF2-OF3), and additionally red-shifted emission towards NW (OF1). This is seen in $\mathrm{CH}_{3} \mathrm{OH}$, $\mathrm{CH}_{3} \mathrm{CN}\left(12_{4}-11_{4}\right)$, and OCS;

2. transitions with a velocity gradient along the NE-SW axis (OF2-OF3) (HNCO);

3. transitions with a velocity gradient along the E-W axis (high excitation $\mathrm{CH}_{3} \mathrm{CN}$ lines).

As seen before, the first case can be explained by a combination of the blue- and red-shifted lobes of the OF1, OF2-OF3 outflows, indicating for $\mathrm{CH}_{3} \mathrm{OH}, \mathrm{CH}_{3} \mathrm{CN}\left(12_{4}-11_{4}\right)$, and OCS at least a non negligible contamination from the multiple outflows in the IRAS 17233-3606 cluster.

While the association of $\mathrm{CH}_{3} \mathrm{OH}$ and OCS with molecular outflows is not surprising since they have been detected in several chemical active molecular outflows (e.g., Bachiller \& Pérez Gutiérrez 1997), the detection of a similar behaviour in the $\mathrm{CH}_{3} \mathrm{CN}\left(12_{4}-11_{4}\right)$ transition is unexpected. $\mathrm{CH}_{3} \mathrm{CN}$ is usually considered a pure hot core tracer and is often used to investigate velocity fields around massive YSOs to search for evidence of rotation (see Cesaroni et al. 1999; Beltrán et al. 2004a, 2005, 2011, but also discussion in Gibb et al. 2004 and Araya et al. 2008). However, in the last years Arce et al. (2008) and Codella et al. (2009) detected $\mathrm{CH}_{3} \mathrm{CN}$ towards the molecular outflow driven by the low-mass YSO L1157-mm, hence showing that also this species can be altered by the passage of bow shocks, although with abundances much lower than in hot cores and hot corinos around high- and low-mass YSOs (e.g., Olmi et al. 1993; Beltrán et al. 2005; Bottinelli et al. 2007). Additionally, Zapata et al. (2011) recently proposed that $\mathrm{CH}_{3} \mathrm{CN}$ emission in Orion-KL is also excited by shocks. In the present case, the velocity gradient detected in $\mathrm{CH}_{3} \mathrm{CN}$ could be due to outflowenvelope interactions as already seen in low-mass star class 0 sources (Beltrán et al. 2004b; Arce \& Sargent 2005; Lee et al. 2005 ) in other molecular species.

The second kinematical pattern (HNCO) can be easily associated with the motions of the OF2-OF3 outflows. HNCO is abundant in densest regions of molecular clouds (Jackson et al. 1984; Wyrowski et al. 1999) as well as in regions of low velocity shocks (Martín et al. 2008). However, very recently also HNCO has been detected towards the chemical active L1157 flow by Rodríguez-Fernández et al. (2010), showing that the HNCO line profiles exhibit the same characteristics as other well-known tracers of shocked gas and supporting the idea that HNCO is a good tracer of interstellar shocks. Our data support this scenario, reporting the first detection of $\mathrm{HNCO}$ emission in outflows driven by massive YSOs.

The third case (velocity gradient almost perpendicular to OF2-OF3) is the most intriguing since $\mathrm{CH}_{3} \mathrm{CN}$ emission has been extensively used in the last years to trace rotating toroids supposed to host accretion disks around massive YSOs. This has been used to support a mechanism for the formation of massive stars similar to that of low-mass stars. In the case of IRAS 17233, at least three possible interpretations can be given for the E-W velocity gradient detected in the $\mathrm{CH}_{3} \mathrm{CN}$ lines: rotation, contamination by outflows, velocity differences between cores.

In the first case, the E-W velocity gradient could trace a rotating structure perpendicular to OF2-OF3, the dominant outflow of this region. In this case, following Beltrán et al. (2004a, 2005) we computed the dynamical mass $M_{\text {dyn }}$ assuming equilibrium between centrifugal and gravitational forces. We took as rotation velocity $V_{\text {rot }}=3 \mathrm{~km} \mathrm{~s}^{-1}$, value corresponding to half the velocity range measured in the $\mathrm{CH}_{3} \mathrm{CN}\left(12_{4}-11_{4}\right)$ line 


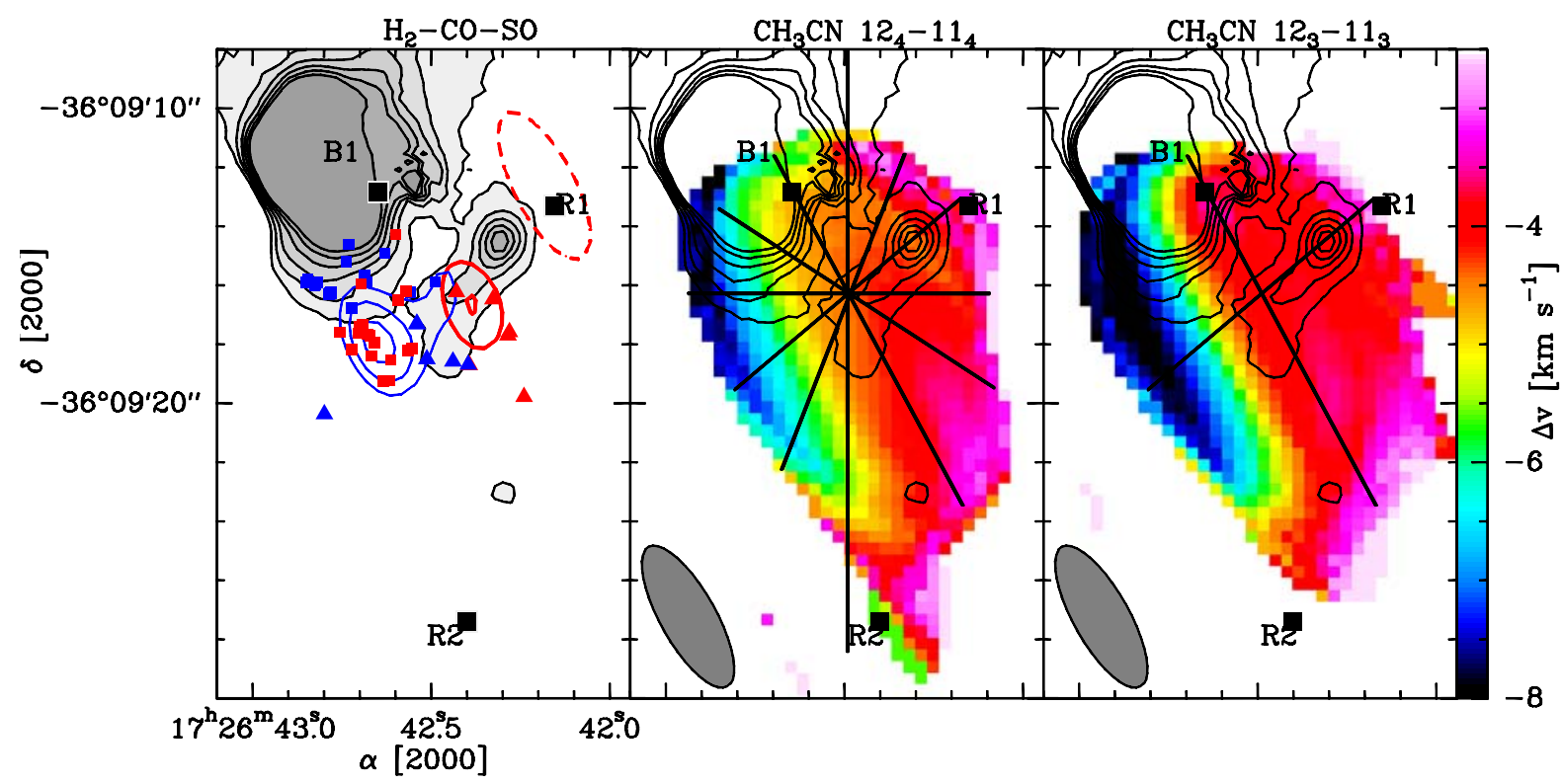

Fig. 9. Summary of the high angular resolution observations available for the IRAS 17233 cluster (see previous figures for details and references), Left: maps of the $\mathrm{H}_{2}$ emission (grey and solid contour levels). The blue and red contours represent the $\mathrm{SO}\left(5_{6}-4_{5}\right)$ blue- and red-shifted emission; the dashed red contour is the $\mathrm{CO}(2-1)$ red-shifted emission at extremely high velocities. Overlaid on the map are also the positions of the blue and red $\mathrm{OH}$ (squares) and $\mathrm{H}_{2} \mathrm{O}$ (triangles) masers. Middle: overlay of the $\mathrm{CH}_{3} \mathrm{CN}\left(12_{4}-11_{4}\right)$ first moment map (colour scale) with the $\mathrm{H}_{2}$ emission map (black contours). The black lines represent the directions used in the position-velocity plots of Fig. 12 and are: the OF1 axis; the OF2-OF3 axis (NE-SW line); the N-S and E-W directions; the cuts with position angles of -20 and $60^{\circ}$. Right: overlay of the $\mathrm{CH}_{3} \mathrm{CN}\left(12_{3}-11_{3}\right)$ first moment map (colour scale) with the $\mathrm{H}_{2}$ emission map (black contours). The black lines represent the axes of the OF1 and OF2-OF3 outflows. The R1, R2 and $\mathrm{B} 1$ positions in $\mathrm{OF} 1$ and $\mathrm{OF} 2-\mathrm{OF} 3$ respectively are marked by black squares.

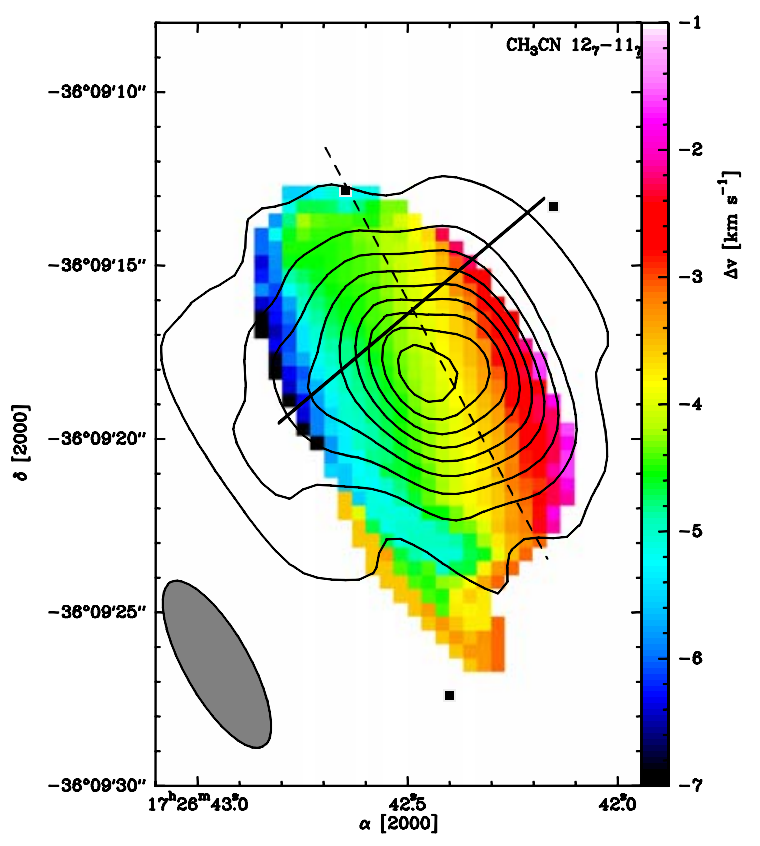

Fig. 10. First moment map of the $\mathrm{CH}_{3} \mathrm{CN}\left(12_{7}-11_{7}\right)$ line. The black solid contours show the $1.3 \mathrm{~mm}$ continuum emission. Black squares mark the B1, R1 and R2 positions of Fig. 1a. The other symbols are as in Fig. 4. The systemic velocity of the source lies between -3.4 and $-2.5 \mathrm{~km} \mathrm{~s}^{-1}$ (see Sect. 5.2).

and as radius half the deconvolved $F W H M$ size of the continuum emission ( $S=5^{\prime \prime} .3$, see Table 3 ). This corresponds to a dynamical mass of $27 M_{\odot}$ for an edge-on toroid. This is likely a lower limit to the real dynamical mass since the inclination

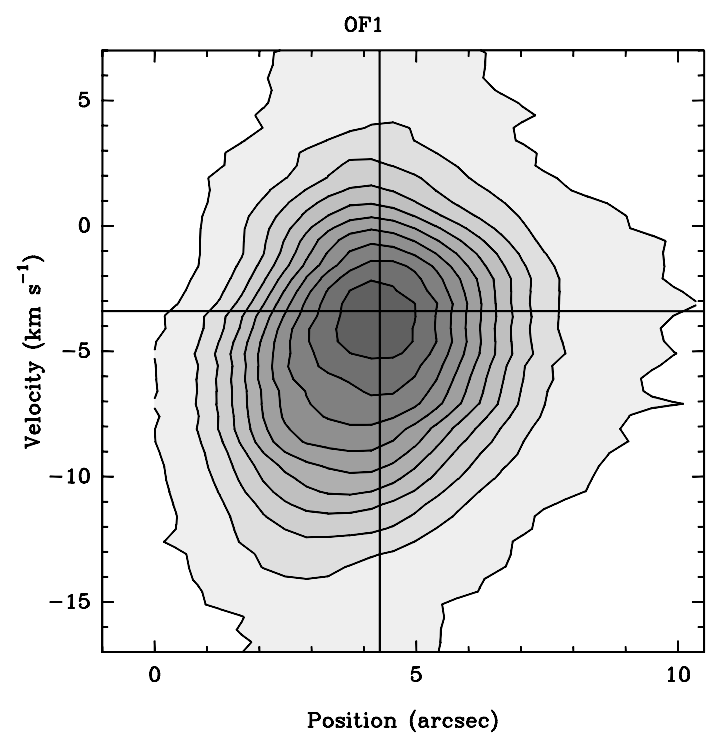

Fig. 11. Position-velocity plot of the $\mathrm{CH}_{3} \mathrm{CN}\left(12_{4}-11_{4}\right)$ line along the OF1 axis. Contour levels range from $5 \sigma\left(0.5 \mathrm{Jy}\right.$ beam $\left.^{-1}\right)$ in step of $20 \sigma$. The horizontal line marks the systemic velocity of $-3.4 \mathrm{~km} \mathrm{~s}^{-1}$, the vertical line the crossing point of all slits.

angle is unknown. Such value is consistent with those estimated by Beltrán et al. (2004a, 2011) for other sources.

However, contamination by outflows questions this interpretation, since non negligible velocity gradients are detected along the OF2-OF3 axis and along other directions (see Sect. 5.2 and Fig. 12). In this context, the velocity gradient along the E$\mathrm{W}$ direction could be due to a composition of motions along 


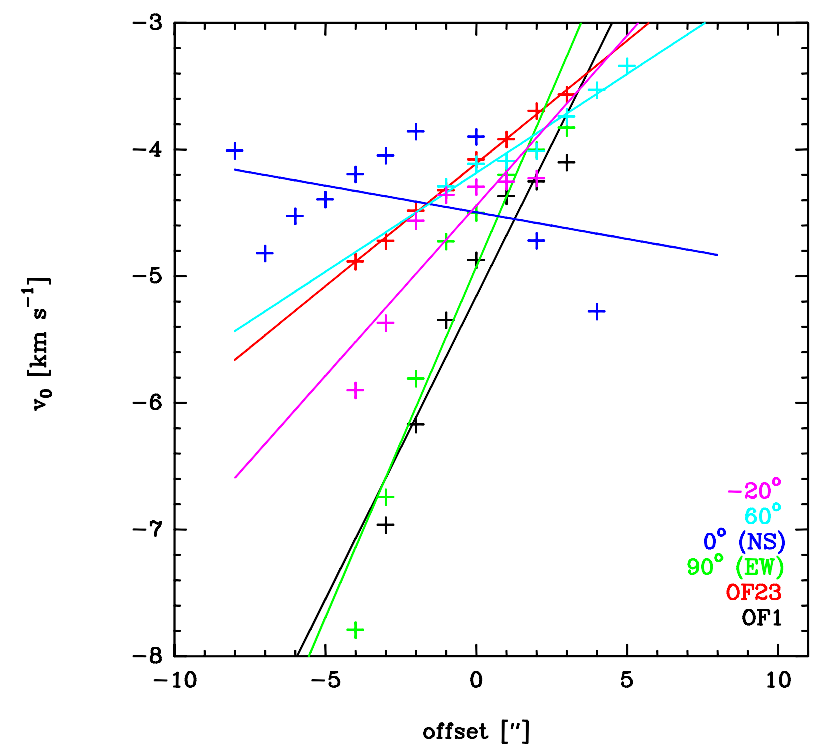

Fig. 12. Peak velocity of the of $\mathrm{CH}_{3} \mathrm{CN}\left(12_{4}-11_{4}\right)$ line along six different directions outlined in Fig. 9 as function of the offset position along the slit. Different colours denote different slits. The solid lines represent the fit to the data.

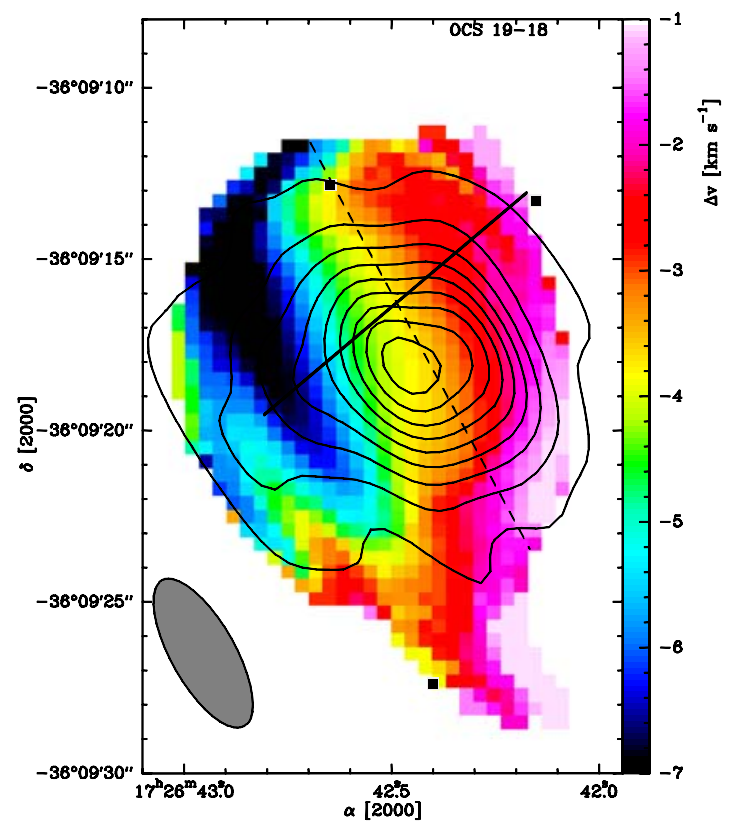

Fig. 13. First moment map of the OCS(19-18) transition. The black solid contours show the $1.3 \mathrm{~mm}$ continuum emission. Black squares mark the B1, R1 and R2 positions of Fig. 1a. The other symbols are as in Fig. 4. The systemic velocity of the source lies between -3.4 and $-2.5 \mathrm{~km} \mathrm{~s}^{-1}$ (see Sect. 5.2).

OF2-OF3 and OF1. Finally, since four cm compact VLAsources are in the beam of our SMA data, and they are roughly located along the E-W axis, one could consider that the kinematical patterns here reported are simply due to a velocity difference of the sources.

Clearly, higher angular observations are needed to discriminate between these interpretations. Given its low declination, ALMA is the ideal instrument to investigate the source at higher resolution.

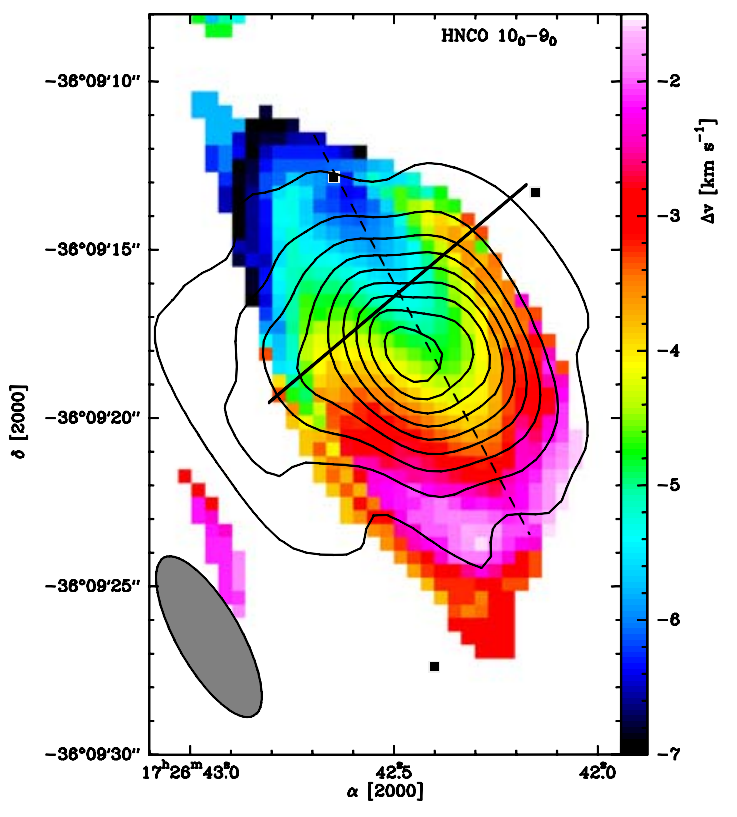

Fig. 14. First moment maps of the $\operatorname{HNCO}\left(10_{0}-9_{0}\right)$ line. The black solid contours show the $1.3 \mathrm{~mm}$ continuum emission. Black squares mark the B1, R1 and R2 positions of Fig. 1a. The other symbols are as in Fig. 4. The systemic velocity of the source lies between -3.4 and $-2.5 \mathrm{~km} \mathrm{~s}^{-1}$ (see Sect. 5.2).

\section{Implications for studies of high-mass YSOs}

Although not conclusive for the search of tracers of rotation around massive YSOs, we believe that our study of IRAS 17233 has important implications for searches of disks in high-mass star forming regions. IRAS 17233 is an exceptionally close and active site of massive star formation. Therefore, even observations with a limited angular resolution allow us to resolve small linear scales. Since the distance of the source is $D \leq 1 \mathrm{kpc}$, our beam of $\sim 5.4^{\prime \prime} \times 2^{\prime \prime}$ corresponds to a maximum linear scale of $5400 \times 2000 \mathrm{AU}$, thus comparable with state-of-the-art interferometric observations of more distant massive YSOs. For example, the recent study of Beltrán et al. (2011) was performed with an linear resolution between 2400 and 50000 AU.

To verify whether the detection of OF1 in the $\mathrm{CH}_{3} \mathrm{CN}\left(12_{4}-11_{4}\right)$ transition would have been possible with lower linear resolution (i.e., a more distant object), we smoothed our data cube of a factor of two in spatial resolution. This corresponds to observations of a massive YSO at a distance twice as large as that of IRAS 17233, but with the same angular resolution as our observations of IRAS 17233. We then computed moment maps for the $\mathrm{CH}_{3} \mathrm{CN}\left(12_{4}-11_{4}\right)$ transition following the same procedure described in Sect. 5. We also smoothed the $\mathrm{CO}(2-1)$ data cube to the same angular resolution, and derived integrated intensity maps for the blue- and red-shifted emission in the same velocity range as in Fig. 1. The resulting maps are presented in Fig. 15.

The data at lower angular resolution show a compact region detected in $\mathrm{CH}_{3} \mathrm{CN}$ with a velocity gradient perpendicular to the dominant outflow of the region, OF2-OF3. Clearly, the red-shifted emission along the OF1 axis is not longer detected, and the derived velocity field is very similar to that detected for the $\mathrm{CH}_{3} \mathrm{CN}(\mathrm{K}=7,8)$ lines. In other words the picture of Fig. 15 could easily be explained with a pure velocity gradient perpendicular to the $\mathrm{N}-\mathrm{S}$ outflow as seen in $\mathrm{CO}$. We note that for sources at distances $\geq 10 \mathrm{kpc}$ also the detection of $\mathrm{H}_{2}$ 


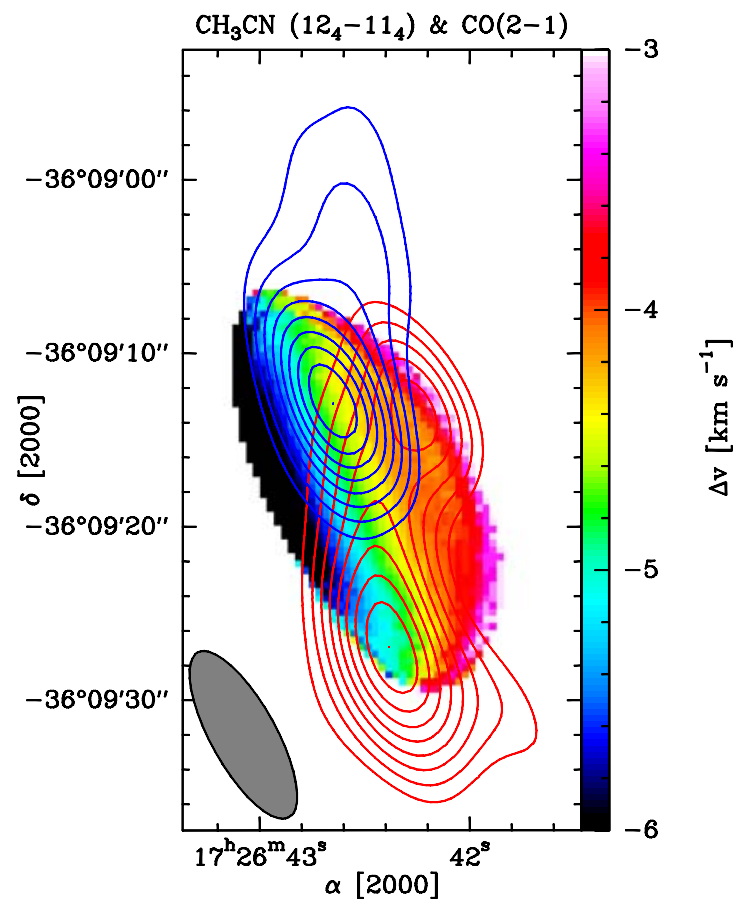

Fig. 15. Velocity gradient of the $\mathrm{CH}_{3} \mathrm{CN}\left(12_{4}-11_{4}\right)$ transition smoothed to an angular resolution of 10 ' $86 \times 3$ '. 78 . The red and blue solid contours show the red- and blue-shifted $\mathrm{CO}(2-1)$ emission smoothed to the same angular resolution.

is challenging. Indeed, in the case of IRAS 17233, the $\mathrm{H}_{2}$ data were fundamental to reveal $\mathrm{OF} 1$, since $\mathrm{H}_{2}$ gives a much better picture of the outflow multiplicity than $\mathrm{CO}$ because it traces hot jets and is not contaminated by infalling envelopes and/or swept up cavities.

\section{Conclusions}

In this study, we analysed interferometric line observations of the massive star forming region IRAS 17233 at $230 \mathrm{GHz}$ with an angular resolution of 5'. $4 \times 1$ '. 9 . We studied the spatial distribution and velocity field of different lines and compared them to the distribution of outflows in the region to investigate the nature of the emission. We report the first detection of HNCO in molecular outflows from massive YSOs. Although other interpretations cannot be ruled out, we find that the velocity gradient observed in $\mathrm{CH}_{3} \mathrm{CN}$ could be significantly affected by the OF1 outflow. This follows the recent detection of both molecules in the chemically active outflow L1157, powered by a low-mass YSO. The possible detection of $\mathrm{CH}_{3} \mathrm{CN}$ in molecular outflows questions the choice of the tracer to probe rotating structures around YSOs: one has to keep in mind that the same molecule could be tracing outflows in some objects and rotating disks/toroids in others. Further studies of high-mass star forming regions are needed to verify whether IRAS 17233-3606 represents a standard laboratory where to investigate star formation or whether it is a special case with a too complex scenario. The present observations, with the possible association of $\mathrm{CH}_{3} \mathrm{CN}$ with the $\mathrm{OF} 1$ outflow in a few thousands AU around the peak of the continuum, stress the importance of (i) very high-spatial resolution, less than $1000 \mathrm{AU}$, and of (ii) $\mathrm{H}_{2}$ images for detailed studies of kinematics in regions as those around massive YSOs. Assuming typical distances of massive star forming regions between 5 and $10 \mathrm{kpc}$, a resolution of $1000 \mathrm{AU}$ corresponds to an angular size of $0{ }^{\prime} 1-0.2$. Even smaller angular resolutions are then needed to study in details the kinematics of such regions. Only studies of massive YSOs with angular resolution $\leq 0$.' 1 (for distances $>5 \mathrm{kpc}$ ) will clarify whether or not IRAS 17233 represents a special case and will shed light on to the choice of the molecular tracers for rotating structures.

As a final remark, given the chemical differences in hot cores, and contamination by outflow emission, potentially in all species, it well may be the case that no molecular species or transition will work for every source under every circumstance. However, we can try to formulate criteria for convincing detection of a rotating disk/toroid in millimeter molecular emission: (i) such a detection should be based on the analysis of high excitation multiple lines and multiple species; (ii) the lines used in the analysis should be unblended; (iii) any possible contribution from outflows should be ruled out; (iv) all lines should be consistent with a single velocity field of a rotating body. As noted in the previous section, selective tracers of hot jets as $\mathrm{H}_{2}$, or $\mathrm{SiO}$, are better suited to understand the multiplicity and morphology of outflows than $\mathrm{CO}$ and may therefore better help to understand the kinematics of the cores.

Acknowledgements. We are grateful to M. Walmsley and A. Gusdorf for fruitful discussions and suggestions. We would also like to thank an anonymous referee for a careful review of the manuscript.

\section{References}

Araya, E., Hofner, P., Kurtz, S., Olmi, L., \& Linz, H. 2008, ApJ, 675, 420 Arce, H. G., \& Sargent, A. I. 2005, ApJ, 624, 232

Arce, H. G., Santiago-García, J., Jørgensen, J. K., Tafalla, M., \& Bachiller, R. 2008, ApJ, 681, L21

Bachiller, R., \& Pérez Gutiérrez, M. 1997, ApJ, 487, L93

Beltrán, M. T., Cesaroni, R., Neri, R., et al. 2004a, ApJ, 601, L187

Beltrán, M. T., Gueth, F., Guilloteau, S., \& Dutrey, A. 2004b, A\&A, 416, 631

Beltrán, M. T., Cesaroni, R., Neri, R., et al. 2005, A\&A, 435, 901

Beltrán, M. T., Cesaroni, R., Neri, R., \& Codella, C. 2011, A\&A, 525, A151

Beuther, H., Schilke, P., Menten, K. M., et al. 2002, ApJ, 566, 945

Beuther, H., Hunter, T. R., Zhang, Q., et al. 2004, ApJ, 616, L23

Beuther, H., Schilke, P., Menten, K. M., et al. 2005a, ApJ, 633, 535

Beuther, H., Zhang, Q., Sridharan, T. K., \& Chen, Y. 2005b, ApJ, 628, 800

Beuther, H., Walsh, A. J., \& Longmore, S. N. 2009, ApJS, 184, 366

Bonnell, I. A., Larson, R. B., \& Zinnecker, H. 2007, Protostars and Planets V, 149

Bottinelli, S., Ceccarelli, C., Williams, J. P., \& Lefloch, B. 2007, A\&A, 463, 601 Brand, J., \& Blitz, L. 1993, A\&A, 275, 67

Bronfman, L., Nyman, L.-A., \& May, J. 1996, A\&AS, 115, 81

Caswell, J. L., Haynes, R. F., \& Phys, J. 1980, IAU Circ., 3509, 2

Cesaroni, R., Felli, M., Testi, L., Walmsley, C. M., \& Olmi, L. 1997, A\&A, 325, 725

Cesaroni, R., Felli, M., Jenness, T., et al. 1999, A\&A, 345, 949

Cesaroni, R., Galli, D., Lodato, G., Walmsley, C. M., \& Zhang, Q. 2007, Protostars and Planets V, 197

Codella, C., Benedettini, M., Beltrán, M. T., et al. 2009, A\&A, 507, L25

Comito, C., Schilke, P., Phillips, T. G., et al. 2005, ApJS, 156, 127

Faúndez, S., Bronfman, L., Garay, G., et al. 2004, A\&A, 426, 97

Fish, V. L., Reid, M. J., Argon, A. L., \& Zheng, X.-W. 2005, ApJS, 160, 220

Fix, J. D., Mutel, R. L., Gaume, R. A., \& Claussen, M. J. 1982, ApJ, 259, 657

Forster, J. R., \& Caswell, J. L. 1989, A\&A, 213, 339

Gibb, A. G., Wyrowski, F., \& Mundy, L. G. 2004, ApJ, 616, 301

Hughes, V. A., \& MacLeod, G. C. 1993, AJ, 105, 1495

Jackson, J. M., Armstrong, J. T., \& Barrett, A. H. 1984, ApJ, 280, 608

Kraus, S., Hofmann, K., Menten, K. M., et al. 2010, Nature, 466, 339

Krumholz, M. R., Klein, R. I., McKee, C. F., Offner, S. S. R., \& Cunningham, A. J. 2009, Science, 323, 754

Kuiper, R., Klahr, H., Beuther, H., \& Henning, T. 2010, ApJ, 722, 1556

Lee, C., Ho, P. T. P., \& White, S. M. 2005, ApJ, 619, 948 
S. Leurini et al.: On the kinematics of massive star forming regions: the case of IRAS 17233-3606

Leurini, S., Codella, C., Zapata, L. A., et al. 2009, A\&A, 507, 1443

Leurini, S., Pillai, T., Stanke, T., et al. 2011, A\&A, submitted

MacLeod, G. C., Scalise, E. J., Saedt, S., Galt, J. A., \& Gaylard, M. J. 1998, AJ, 116, 1897

Martín, S., Requena-Torres, M. A., Martín-Pintado, J., \& Mauersberger, R. 2008, ApJ, 678, 245

McKee, C. F., \& Tan, J. C. 2002, Nature, 416, 59

McKee, C. F., \& Tan, J. C. 2003, ApJ, 585, 850

Menten, K. M. 1991, ApJ, 380, L75

Miettinen, O., Harju, J., Haikala, L. K., \& Pomrén, C. 2006, A\&A, 460, 721

Müller, H. S. P., Thorwirth, S., Roth, D. A., \& Winnewisser, G. 2001, A\&A, 370, L49

Müller, H. S. P., Schlöder, F., Stutzki, J., \& Winnewisser, G. 2005, Mol. Struct., 742,215
Olmi, L., Cesaroni, R., \& Walmsley, C. M. 1993, A\&A, 276, 489

Pickett, H. M., Poynter, I. R. L., Cohen, E. A., et al. 1998, Quant. Spect. Rad. Transf., 60, 883

Rodríguez-Fernández, N. J., Tafalla, M., Gueth, F., \& Bachiller, R. 2010, A\&A, 516, A98

Walsh, A. J., Burton, M. G., Hyland, A. R., \& Robinson, G. 1998, MNRAS, 301 640

Wyrowski, F., Schilke, P., Walmsley, C. M., \& Menten, K. M. 1999, ApJ, 514, L43

Zapata, L. A., Leurini, S., Menten, K. M., et al. 2008, AJ, 136, 1455

Zapata, L. A., Ho, P. T. P., Schilke, P., et al. 2009, ApJ, 698, 1422

Zapata, L. A., Tang, Y., \& Leurini, S. 2010, ApJ, 725, 1091

Zapata, L. A., Schmid-Burgk, J., \& Menten, K. M. 2011, A\&A, 529, A24

Zhang, Q., Hunter, T. R., \& Sridharan, T. K. 1998, ApJ, 505, L151 